\title{
Multilinear representations of Free PROs
}

\author{
É. Laugerotte, J.-G. Luque, L. Mignot, F. Nicart
}

March 2, 2018

\begin{abstract}
We describe a structure of PRO on hypermatrices. This structure allows us to define multilinear representations of PROs and in particular of free Pros. As an example of applications, we investigate the relations of the representations of Pros with the theory of automata.
\end{abstract}

Keywords: PROs, Operads, hypermatrices, categories, theory of automata.

\section{Introduction}

The $P R O$ theory is a way to embed an abstract theory of operators into the formalism of categories. Informally, a PRO is a set of operators having several inputs and outputs and which can be composed by branching ones to the others or by juxtaposing them. The name PRO means PROduct category and comes from the interpretation of the juxtaposition as a tensor product. The first occurrence of the notion of PRO dates back from the early works of Mac Lane [23, 24] and was used in a special case by Boardman and Vogt [2, 3] to model homotopy. The PRO theory has many connexions with several fields; sometimes explicitly as in algebraic topology [3] or algebraic combinatorics [6]. Sometimes the connexion was not explicitly identified but the underlying algebraic structure involves naturally a PRO; we give a few examples in the end of the paper (Section 6 appendices $B$ and $C$ ).

As for many algebraic structures, the notion of freeness is well defined for PROs. This means that there exist objects, called free PROs, having the universal property. This property allows to construct onto morphisms from a free PRO to any PRO having the same set of generators. At the other end, we define PROs on hypermatrices. The goal of the paper is to investigate the morphisms from free PROs to PROs on hypermatrices. These morphisms are called multilinear representations.

The paper is organized as follows. Section 2 is devoted to the general study of the notion of PRO. More precisely, we first recall (Section 2.1) the original definition of PRO in category theory. In Section 2.2. we recall the alternative constructive definition. We investigate also several generalizations and variations like colored PRO (Section 2.3) and ModPro (Section 2.4). This last notion allows us to consider some PROs as modules over a semiring. The concept of subPros and quotient are also recalled (see Section 2.5).

Free Pros are defined and studied in Section 3 In particular in Section 2.2, we investigate the case where all the generators have neither empty input nor empty output. Such a free PRO is called Circuit PROs. This is a particularly important example because the elements can be nicely represented by some kind of electronic circuits. We discuss also on the difficulties to find a combinatorial representation for general free PROs (Section 3.2) and we describe a colored PRO on generalized paths on hypergraphs in Section 3.3

The PRO structure on hypermatrices is defined in Section 4.1 and we investigate the properties of the composition and juxtaposition with respect to the Kronecker product (Section 4.2) and the sum (Section 4.3).

This allows us to define multilinear representations of PROs (Section 5.1). We investigate the behavior with respect to the sum on hypermatrices (Section 5.2) and the interpretation in terms of generalized 
paths (Section 5.3).

As an example of applications, in the last section, we investigate the links with the theory of automata 6.

\section{Pros and their generalizations}

This section is devoted to the definitions and the properties of several structures related to the notion of Pro. Pros are bigraded sets of objects (according to the number of inputs and the number of outputs). In Section 2.1 we compare two definitions of Pros which can be found in literature. The first one comes directly from the category theory and the second is more combinatorial.

\subsection{What PROs are}

In modern algebra, $P R O$ s are defined in category theory as strict monoidal categories whose objects are the natural numbers (including zero), and whose tensor product is the addition. Before giving a more combinatorial way to define PROs, let us explain what this first definition means. First we recall that the aim of category theory is to provide tools to describe in an abstract way classes of mathematical objects (sets, monoids, algebras, etc.). A class is encoded by a graph whose vertices are the objects and whose arrows encode the morphisms. More precisely, a category $C$ is constituted with three entities:

- The class $\operatorname{Obj}(C)$ of its objects,

- The class $\operatorname{Hom}(C)$ of its arrows (also called morphisms). Each arrow has a source and a target which belong to $\operatorname{Obj}(C)$. The set of the arrows whose source is $a$ and whose target is $b$ is denoted by $\operatorname{Hom}_{C}(a, b)$ (or $\operatorname{Hom}(a, b)$ when there is no ambiguity).

- A binary operation $\circ$, called composition such that for any $a, b, c \in \operatorname{Obj}(C), \circ: \operatorname{Hom}(b, c) \times$ $\operatorname{Hom}(a, b) \rightarrow \operatorname{Hom}(a, c)$. When there is no ambiguity, we omit to write $\circ$. The composition must satisfy two properties,

- Associativity. $f(g h)=(f g) h$

- Identity. For any object $a \in \operatorname{Obj}(C)$, there exists a unique morphism $1_{a} \in \operatorname{Hom}(a, a)$ called identity on $a$. These arrows satisfy $1_{b} f=f 1_{a}=f$ for any $f \in \operatorname{Hom}(a, b)$. When there is no ambiguity the identity is simply denoted by 1 .

Functors are morphisms between categories which encode maps preserving identity and compositions. Let $C$ and $\mathcal{D}$ be two categories. A functor $F$ from $C$ to $\mathcal{D}$ is a map that associates to each object $a \in C$ an object $F(a) \in \mathcal{D}$ and to each arrow $f \in \operatorname{Hom}(a, b)$ an arrow $F(f) \in \operatorname{Hom}(F(a), F(b))$ such that the image of the $1_{a}$ is $1_{F(a)}$ and $F(f \circ g)=F(f) \circ F(g)$. If $F$ and $G$ are functors between two categories $C$ and $\mathcal{D}$, a natural transformation $\eta$ is a family of morphisms such that for each object $a \in C$ there exists a morphism $\eta_{a}: F(a) \rightarrow G(a)$ in $\mathcal{D}$ called component of $\eta$ at $a$ satisfying $\eta_{b} \circ F(f)=G(f) \circ \eta_{a}$ for every morphism $f: a \rightarrow b$. In the aim to simplify the notation, we use $\eta$ instead of $\eta_{a}$ when there is no ambiguity.

The product category $C \times \mathcal{D}$ is the category whose objects are the pairs $(a, b)$ with $a \in C$ and $b \in \mathcal{D}$ and whose arrows are the pairs of morphisms $(f, g)$ such that $f \in \operatorname{Hom}\left(a, a^{\prime}\right)$ and $g \in \operatorname{Hom}\left(b, b^{\prime}\right)$; the composition is the component-wise composition. More precisely,

$$
(f, g) \circ\left(f^{\prime}, g^{\prime}\right)=\left(f \circ f^{\prime}, g \circ g^{\prime}\right) \text { and } 1_{(a, b)}=\left(1_{a}, 1_{b}\right) \text {. }
$$

A monoidal category is a category $\mathcal{M}$ equiped with a (bi)functor $\otimes: \mathcal{M} \times \mathcal{M} \rightarrow \mathcal{M}$, an object $I$ called the unit object or the identity object, three natural isomorphisms

1. the associator $\alpha$ with components $\alpha_{a, b, c}:(a \otimes b) \otimes c \simeq a \otimes(b \otimes c)$; 
2. the left unitor $\lambda$ with components $\lambda_{a}: I \otimes a \simeq a$,

3. the right unitor $\rho$ with components $\rho_{a}: a \otimes I \simeq a$

satisfying $(1 \otimes \alpha) \alpha(\alpha \otimes 1)=\alpha \alpha:((a \otimes b) \otimes c) \otimes d) \rightarrow a \otimes(b \otimes(c \otimes d))$ and $(1 \otimes \lambda) \alpha=\rho \otimes 1:(a \otimes 1) \otimes b \rightarrow a \otimes b$ for any objects $a, b, c, d$ in $\mathcal{M}$.

A monoidal category $(\mathcal{M}, \alpha, \lambda, \rho, I)$ is said strict if the natural isomorphisms $\alpha, \lambda$ and $\rho$ are identities. In other words

$$
a \otimes(b \otimes c)=(a \otimes b) \otimes c
$$

for any $a, b, c \in \operatorname{Obj}(\mathcal{M})$,

$$
f \otimes(g \otimes h)=(f \otimes g) \otimes h
$$

for any $f, g, h \in \operatorname{Hom}(\mathcal{M}), I \otimes a=a=a \otimes I$ for any $a \in \operatorname{Obj}(\mathcal{M})$, and $1 \otimes f=f \otimes I=f$ for any $f \in \operatorname{Hom}(\mathcal{M})$ (i.e. the left and right multiplication by $I$ are the identity functor). Notice that, for any $f \in \operatorname{Hom}\left(a, a^{\prime}\right)$ and $g \in \operatorname{Hom}\left(b, b^{\prime}\right), f \otimes g$ is an arrow whose source is $a \otimes b$ and target is $a^{\prime} \otimes b^{\prime}$. Furthermore from (1), we have an additional identity :

$$
(f \circ g) \otimes\left(f^{\prime} \circ g^{\prime}\right)=\left(f \otimes f^{\prime}\right) \circ\left(g \otimes g^{\prime}\right)
$$

for any $f \in \operatorname{Hom}(a, b), g \in \operatorname{Hom}(b, c), f^{\prime} \in \operatorname{Hom}\left(a^{\prime}, b^{\prime}\right)$, and $g^{\prime} \in \operatorname{Hom}\left(b^{\prime}, c^{\prime}\right)$

A $P R O \mathcal{P}$ is a strict monoidal category whose object are the natural numbers and the tensor product sends $(m, n)$ to $m+n$. Hence, if $f \in \operatorname{Hom}(m, n)$ and $g \in \operatorname{Hom}\left(m^{\prime}, n^{\prime}\right)$ then we have $f \otimes g \in$ $\operatorname{Hom}\left(m+m^{\prime}, n+n^{\prime}\right)$. Obviously, the unit object of the category is 0 .

Example 1. Suppose that $\operatorname{Hom}(m, n)=\left\{f_{m, n}\right\}$. In other words, the morphisms are the edges of the complete graph whose vertices are the integers. In addition, we define a composition $\circ$ satisfying $f_{m, n} \circ f_{n, p}=f_{m, p}$ for any integers $n, m, p \in \mathbb{N}$. Obviously, this defines a category. Now, if we set $f_{m, n} \otimes f_{m^{\prime}, n^{\prime}}=f_{m+m^{\prime}, n+n^{\prime}}$, then the category is endowed with a structure of PRO.

Example 2. The category FinSet, whose objects are all finite sets and whose morphisms are all functions between them, is a PRO. Each integer $n$ is identified with a unique set $\{0, \ldots, n-1\}$ and a morphism from $m$ to $n$ is a $m$-tuple $\left(\alpha_{0}, \ldots, \alpha_{m-1}\right)$ such that $0 \leq \alpha_{i} \leq n-1$ for each $0 \leq i \leq m-1$.

This definition being very formal, some properties, like formula (2), are implicit. Furthermore we have

$$
1_{n}=\overbrace{1_{1} \otimes \cdots \otimes 1_{1}}^{n \text { times }}
$$

for $n>0$ as a consequence of $1_{m} \otimes 1_{n}=1_{m+n}$ and formula (1), and $1_{0}$ is the identity on 0 . The last equality is a consequence of formula (2).

In the next section, we give an alternative combinatorial definition and make a parallel with the first one. Nevertheless, the algebraicity can be used to propose structures naturally derived from the notion of PRO. In our paper, we will use two kinds of processes. The first one consists in endowing each $\operatorname{Hom}(a, b)$ with an additional algebraic structure and some compatibility conditions. The second one consists in changing the objects in the strict monoidal category. For instance, we will investigate the notion of colored PROs which are strict monoidal categories whose objects are vectors of colours and whose tensor product is the catenation of the vectors.

\subsection{A combinatorial definition for PROs}

In this section, we give an alternative definition for PROs used in the context of algebraic combinatorics (see e.g. [6]).

A $P R O$ is a bi-graded set $\mathcal{P}=\bigcup_{m, n \in \mathbb{N}} \mathcal{P}_{m, n}$ endowed with two binary operations $\leftrightarrow: \mathcal{P}_{m, n} \times \mathcal{P}_{m^{\prime}, n^{\prime}} \rightarrow$ $\mathcal{P}_{m+m^{\prime}, n+n^{\prime}}$ (horizontal composition) and $\uparrow: \mathcal{P}_{m, n} \times \mathcal{P}_{n, p} \rightarrow \mathcal{P}_{m, p}$ (vertical composition) which satisfy the following rules: 
- Horizontal associativity. For any $\mathfrak{p}, \mathfrak{p}^{\prime}, \mathfrak{p}^{\prime \prime} \in \mathcal{P}$, we have:

$$
\mathfrak{p} \leftrightarrow\left(\mathfrak{p}^{\prime} \leftrightarrow \mathfrak{p}^{\prime \prime}\right)=\left(\mathfrak{p} \leftrightarrow \mathfrak{p}^{\prime}\right) \leftrightarrow \mathfrak{p}^{\prime \prime} .
$$

- Vertical associativity. For each $\mathfrak{p} \in \mathcal{P}_{m, n}, \mathfrak{q} \in \mathcal{P}_{n, p}$ and $\mathfrak{r} \in \mathcal{P}_{p, q}$ we have

$$
\left(\begin{array}{l}
\mathfrak{p} \\
\mathfrak{q} \\
\mathfrak{l} \\
\mathfrak{r}
\end{array}\right)=\left(\begin{array}{l}
\mathfrak{p} \\
\mathfrak{l} \\
\mathfrak{q}
\end{array}\right)
$$

- Interchange law. For each $\mathfrak{p} \in \mathcal{P}_{m, n}, \mathfrak{q} \in \mathcal{P}_{n, p}, \mathfrak{p}^{\prime} \in \mathcal{P}_{m^{\prime}, n^{\prime}}$ and $\mathfrak{q}^{\prime} \in \mathcal{P}_{n^{\prime}, p^{\prime}}$ we have

$$
\left(\begin{array}{l}
\mathfrak{p} \\
\mathfrak{l} \\
\mathfrak{q}
\end{array}\right) \leftrightarrow\left(\begin{array}{c}
\mathfrak{p}^{\prime} \\
\uparrow \\
\mathfrak{q}^{\prime}
\end{array}\right)=\begin{gathered}
\left(\mathfrak{p} \leftrightarrow \mathfrak{p}^{\prime}\right) \\
\left(\mathfrak{q} \leftrightarrow \mathfrak{q}^{\prime}\right) .
\end{gathered}
$$

- Graded vertical unit. For each $n \in \mathbb{N}$ there exists a unique graded unit $I d_{n}$. More precisely, one has

$$
\begin{gathered}
I d_{m} \\
\mathfrak{l} \\
\mathfrak{p}
\end{gathered}=\begin{gathered}
\mathfrak{p} \\
I d_{n}
\end{gathered}=\mathfrak{p}
$$

for each $\mathfrak{p} \in \mathcal{P}_{m, n}$. Moreover we must have

$$
I d_{n}=I d_{1}^{\leftrightarrow n}=\overbrace{I d_{1} \leftrightarrow \cdots \leftrightarrow I d_{1}}^{n \text { times }}
$$

for $n \geq 1$.

- Horizontal unit. There exists an element $\varepsilon$ such that for each $\mathfrak{p} \in \mathcal{P}$ we have

$$
\varepsilon \leftrightarrow \mathfrak{p}=\mathfrak{p} \leftrightarrow \varepsilon=\mathfrak{p} .
$$

For simplicity, when there is no ambiguity, we denote $I d_{1}=\mid, I d_{n}=\overbrace{|\cdots|}^{n}$ and $I d_{0}=\oslash$. Observe that $(\mathcal{P}, \leftrightarrow)$ is a monoid whose unit is $\oslash$ and each $\left(\mathcal{P}_{n, n}, \uparrow\right)$ is a monoid whose unit is $\overbrace{|\cdots|}^{n}$. Each element $p \in \mathcal{P}_{m, n}$ with $m, n>0$ will be called pluggable.

Naturally, morphism 1 of PROs are defined as maps $\phi: \mathcal{P} \rightarrow Q$ that carry the algebraic structures. More precisely, a morphism $\phi$ satisfies the following properties:

- Graded map.

$$
\phi\left(\mathcal{P}_{m, n}\right) \subset Q_{m, n}
$$

- Morphism of monoid.

$$
\phi(\mathfrak{p} \leftrightarrow \mathfrak{q})=\phi(\mathfrak{p}) \leftrightarrow \phi(\mathfrak{q}) .
$$

- Compatibility with the vertical composition.

$$
\phi\left(\begin{array}{l}
\mathfrak{p} \\
\mathfrak{\downarrow} \\
\mathfrak{q}
\end{array}\right)=\begin{gathered}
\phi(\mathfrak{p}) \\
\uparrow \\
\phi(\mathfrak{q})
\end{gathered} .
$$

\footnotetext{
${ }^{1}$ In the algebraic definition, these morphisms are monoidal functors.
} 
- The image of a unit is a unit.

$$
\phi(\overbrace{|\cdots|}^{n})=\overbrace{|\cdots|}^{n}
$$

for each $n \in \mathbb{N}$.

Let us compare the two definitions:

\begin{tabular}{|c|c|}
\hline Combinatorial definition & Categorial definition \\
\hline $\mathcal{P}_{m, n}$ & $\operatorname{Hom}(m, n)$ \\
Vertical composition & Composition of the morphisms \\
Horizontal composition & Tensor product \\
$I d_{n}$ & Identity on $n$ \\
$\varepsilon$ & Identity on the unit object \\
Morphism & Monoidal functor \\
\hline
\end{tabular}

Notice that the interchange law and the equality $I d_{n}=I d_{1}^{\leftrightarrow n}$ are now axioms whilst they are deduced from the strict monoidal structure in the first definition. Also we have

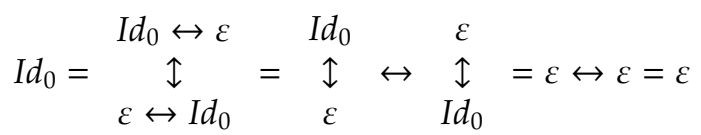

Hence, the horizontal unit is unique: $\varepsilon=I d_{0}$ as for the algebraic definition.

\subsection{Colored PROs}

The notion of colored PRO extends the notion of PRO to more general graded sets with finer graduation. Consider a set $\mathbf{C}$ of colors. A colored PRO is a graded set

$$
\mathcal{P}=\bigcup_{n, m \geq 0} \bigcup_{\substack{I \subset C^{n} \\ I \subset C^{m}}} \mathcal{P}_{I, J}
$$

endowed with two laws $\leftrightarrow: \mathcal{P}_{I, J} \times \mathcal{P}_{I^{\prime}, J^{\prime}} \longrightarrow \mathcal{P}_{I I^{\prime}, J J^{\prime}}$, where $I I^{\prime}$ is the catenation of the lists $I$ and $I^{\prime}$ and $\uparrow: \mathcal{P}_{I, J} \times \mathcal{P}_{J, K} \longrightarrow \mathcal{P}_{I, K}$ satisfying the horizontal associativity, the vertical associativity, and the interchange law and such that

- Graded vertical unit: For each $n \geq 0$ and $I \in \mathbf{C}^{n}$ there exists a unique element $I d_{I} \subset \mathcal{P}_{I, I}$ verifying

$$
\begin{gathered}
I d_{I} \\
\downarrow \\
\mathfrak{p}
\end{gathered}
$$

for each $\mathfrak{p} \in \mathcal{P}_{I, J}$ and

$$
\underset{\mathfrak{q}}{\mathfrak{q}}=\mathfrak{q}
$$

for each $\mathfrak{q} \in \mathcal{P}_{K, I}$. Moreover we must have $I d_{I}=I d_{c_{1}} \leftrightarrow \cdots \leftrightarrow I d_{c_{n}}$ for $I=\left[c_{1} \ldots, c_{n}\right]$.

- Horizontal unit: there exists an element $\varepsilon \in \mathcal{P}_{[],[]}$such that for each $\mathfrak{p} \in \mathcal{P}$ we have

$$
\varepsilon \leftrightarrow \mathfrak{p}=\mathfrak{p} \leftrightarrow \varepsilon=\mathfrak{p} .
$$

As in the case of PRO we have 
Lemma 1. The horizontal unit is unique: $\varepsilon=I d_{[]}$.

In terms of category, a colored PRO is a strict monoidal category such that the objects are vectors of colors and the tensor product sends $\left(\left[c_{1}, \ldots, c_{m}\right],\left[d_{1}, \ldots, d_{n}\right]\right)$ to the catenation $\left[c_{1}, \ldots, c_{m}, d_{1}, \ldots, d_{n}\right]$ of the two vectors. The unit object of the category is the empty vector [].

\subsection{ModPro}

A $\mathbb{K}$-ModPro $\mathrm{M}=\bigcup_{n, m \geq 0} \mathrm{M}_{n, m}$ is a PRO equipped with two additional operations + and $\cdot$ which confer to each $\mathbb{M}_{n, m}$ a structure of $\mathbb{K}$-module satisfying the following rules:

- Left and right distributivities.

$$
\mathrm{p} \leftrightarrow(\mathrm{q}+\mathrm{r})=(\mathrm{p} \leftrightarrow \mathrm{q})+(\mathrm{p} \leftrightarrow \mathrm{r}),(\mathrm{q}+\mathrm{r}) \leftrightarrow \mathrm{p}=(\mathrm{q} \leftrightarrow \mathrm{p})+(\mathrm{r} \leftrightarrow \mathrm{p}),
$$

- Up and down distributivities.

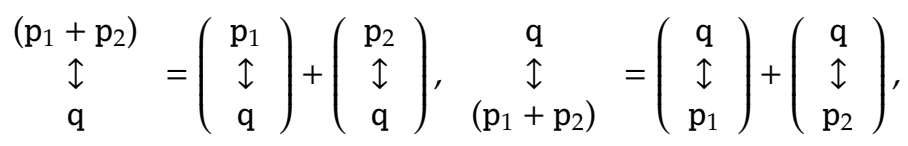

- $a \cdot(\mathrm{p} \leftrightarrow \mathrm{q})=(a \cdot \mathrm{p}) \leftrightarrow \mathrm{q}=\mathrm{p} \leftrightarrow(a \cdot \mathrm{q})$.

- $a \cdot\left(\begin{array}{c}\mathrm{p} \\ \uparrow \\ \mathrm{q}\end{array}\right)=\left(\begin{array}{c}a \cdot \mathrm{p} \\ \uparrow \\ \mathrm{q}\end{array}\right)=\left(\begin{array}{c}\mathrm{p} \\ \uparrow \\ a \cdot \mathrm{q}\end{array}\right)$.

Notice that $\left(\mathrm{M}_{0,0},+, \leftrightarrow\right)$ is a semiring. ModPros can alternatively be defined in terms of enriched categories (see Appendix ).

\subsection{SubPROs and Quotients}

A subPRO of a $\operatorname{PRO} \mathcal{P}$ is a subset $\mathcal{P}^{\prime} \subset \mathcal{P}$ containing any $I d_{n}$ and which is stable for the compositions $\leftrightarrow$ and $\uparrow$.

As for many algebraic structures, there is a notion of quotient of PRO. A congruence $\equiv$ is an equivalence relation which is compatible with the graduation and the two compositions. This means

1. $\mathfrak{p} \equiv \mathfrak{q}$ implies $\mathfrak{p}, \mathfrak{q} \in \mathcal{P}_{m, n}$ for some $m, n \in \mathbb{N}$,

2. $\mathfrak{p} \equiv \mathfrak{p}^{\prime} \in \mathcal{P}_{m, n}, \mathfrak{q} \equiv \mathfrak{q}^{\prime} \in \mathcal{P}_{m^{\prime}, n^{\prime}}$ implies $\mathfrak{p} \leftrightarrow \mathfrak{q} \equiv \mathfrak{p}^{\prime} \leftrightarrow \mathfrak{q}^{\prime}$, and

3. $\mathfrak{p} \equiv \mathfrak{p}^{\prime} \in \mathcal{P}_{m, n}, \mathfrak{q} \equiv \mathfrak{q}^{\prime} \in \mathcal{P}_{n, p}$ implies $\begin{array}{ll}\mathfrak{p} & \begin{array}{l}\mathfrak{p}^{\prime} \\ \mathfrak{q}\end{array} \\ \mathfrak{q} & \mathfrak{l} . \\ \mathfrak{q}^{\prime}\end{array}$.

As a consequence, the quotient set $\mathcal{P} / \equiv=\bigcup_{m, n} \mathcal{P}_{m, n} / \equiv$ inherits a structure of PRO from $\mathcal{P}$. The PRO $\mathcal{P} / \equiv$ is called the quotient of $\mathcal{P}$ by $\equiv$. This also defines a morphism of $\mathrm{PRO} \phi_{\equiv}: \mathcal{P} \rightarrow \mathcal{P} / \equiv$ sending each element on its class.

Conversely, if $\phi: \mathcal{P} \rightarrow Q$ is a morphism of PRO then the equivalence $\equiv_{\phi}$, defined by $\mathfrak{p} \equiv \mathfrak{q}$ if and only if $\phi(\mathfrak{p})=\phi(\mathfrak{q})$, is a congruence and $\mathcal{P} / \equiv_{\phi}$ is isomorphic to $\phi(\mathcal{P}) \subset Q$, the subPRO of $Q$ which is the image of $\mathcal{P}$.

Similarly, one defines quotient of ModPro as a quotient of PRO such that the restriction to each graded component is a quotient of $\mathbb{K}$-module. 


\section{Freeness}

Freeness is a notion generalizing the concept of basis in a vector space to other structures. In category theory, free objects satisfies, when they exist, the universal property. Consider the forgetful (faithful) functor $\mathbf{F}$ sending each PRO to its underlying graded set. Let $\mathcal{X}=\cup_{m, n} \mathcal{X}_{m, n}$ be a bigraded set. A $\mathrm{PRO} \mathcal{P}$ is the free PRO on $\mathcal{X}$ if there exists a canonical bigraded injection $i: \mathcal{X} \rightarrow \mathbf{F}(\mathcal{P})$ satisfying the following universal property: for any $\mathrm{PRO} \mathcal{P}^{\prime}$ and any bigraded map $f: \mathcal{X} \rightarrow \mathbf{F}\left(\mathscr{P}^{\prime}\right)$ there exists a unique morphism of $\operatorname{PRO} \bar{f}: \mathcal{P} \rightarrow \mathcal{P}^{\prime}$ such that $f=\mathbf{F}(g) \circ i$. When it exists, such a PRO is denoted by $\mathcal{F}(\mathcal{X})$. In this case, if a PROP $\mathcal{P}$ is generated by $\mathcal{X}$ then there exists an onto morphism from $\psi: \mathcal{F}(\mathcal{X}) \rightarrow \mathcal{P}$ sending each element $x$ to itself. In other words, $\mathcal{P}$ is isomorphic to the quotient $\mathcal{F}(\mathcal{X}) / \equiv_{\Xi_{\psi}}$.

\subsection{Circuit PROs}

We consider a graded set $C=\bigcup_{n, m \geq 1} C_{n, m}$ such that each $C_{n, m}$ is finite. The elements of $C$ are the chips. Each chip $\mathrm{c} \in C_{n, m}$ is graphically represented by a labeled box with $n$ outputs, drawn from the top and $m$ inputs drawn on the bottom of the box (See in Fig 1 for an example of a $(3,4)$-chip).

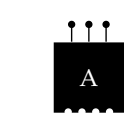

Figure 1: A chip with 3 outputs and 4 inputs

A circuit is an object with outputs and inputs constructed inductively as follow.

Definition 1. A circuit is

1. either the empty circuit denoted by $\oslash$ with 0 output and 0 input,

2. or a a wire denoted by 9 with 1 output and 1 intput,

3. or a chip,

4. or the juxtaposition $\mathfrak{p} \mathfrak{p}^{\prime}$ of two circuits $\mathfrak{p}$ and $\mathfrak{p}^{\prime}$ with the rule $\oslash \mathfrak{p}=\mathfrak{p} \oslash=\mathfrak{p}$. The outputs (resp. the inputs) of $\mathfrak{p} \mathfrak{p}^{\prime}$ is obtained by catening the outputs (resp. the inputs) of $\mathfrak{p}$ and the outputs (resp. the inputs) of $\mathfrak{p}^{\prime}$. We denote by $\leftrightarrow$ the operation of juxtaposition.

5. or the connection of $a(m, n)$-circuit $\mathfrak{p}$ and $a(n, p)$-circuit $\mathfrak{q}$ obtained by connecting the $i$-th input of $\mathfrak{p}$ to the $i$-th output of $q$ with the following two rules:

- connecting $\mathfrak{i} \cdot$ ? to the outputs or the inputs of a circuit let the circuit unchanged,

- connecting $\oslash$ with itself is still $\oslash$.

The outputs of the connection of $\mathfrak{p}$ and $\mathfrak{q}$ are the outputs of $\mathfrak{p}$ and its inputs are the inputs of $\mathfrak{q}$. We denote by $\uparrow$ the operation of connection.

Chips and wires are called elementary circuits.

We denote by $\operatorname{Circ}(C)$ the set of the circuits obtained from the chips of $C$.

Notice that the only circuit having 0 input or 0 output is $\oslash$ and that any non empty circuit can be obtained by juxtaposing and connecting elementary circuits (see Figure 2 for an example).

Straightforwardly from the definition we obtain the following property.

Proposition 1. The operations $\leftrightarrow$ and $\uparrow$ endow $\operatorname{Circ}(C)$ with a structure of PRO. 


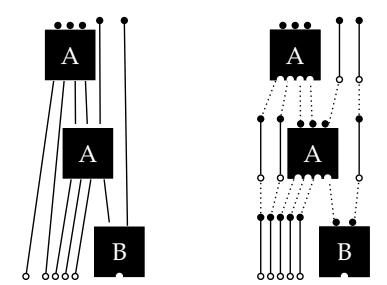

Figure 2: A circuit and a decomposition into elementary circuits

This Pro is known to be isomorphic to $\mathcal{F}(C)$ (see e.g. [6]).

A circuit $\mathfrak{p}$ is deconnected if it is the juxtaposition of two non empty circuits otherwise it is connected. The connected components of $\mathfrak{p}$ is the set of the non-empty connected circuits $\mathfrak{p}_{1}, \ldots, \mathfrak{p}_{k}$ such that

$$
\mathfrak{p}=\mathfrak{p}_{1} \leftrightarrow \mathfrak{p}_{2} \leftrightarrow \cdots \leftrightarrow \mathfrak{p}_{k} .
$$

Proposition 2. Let $\mathfrak{p}$ be a non empty circuit. The following assertions are equivalent:

1. $p$ is connected.

2. $\mathfrak{p}$ has only one connected component.

3. $\mathfrak{p}$ is

- either a chip

- or a wire

- or $\mathfrak{p}$ is a non elementary circuit satisfying

$$
\mathfrak{p}=\begin{gathered}
\left(\mathfrak{p}_{1} \leftrightarrow \cdots \leftrightarrow \mathfrak{p}_{k}\right) \\
\uparrow \\
\left(\mathfrak{q}_{1} \leftrightarrow \cdots \leftrightarrow \mathfrak{q}_{\ell}\right)
\end{gathered}
$$

where $\mathfrak{p}_{i} \in \operatorname{Circ}(C)_{m_{i}, n_{i}}, \mathfrak{q}_{i} \in \operatorname{Circ}(C)_{p_{i}, q_{i}}$ are connected circuits such that $n_{1}+\cdots+n_{i}=p_{1}+\cdots+p_{j}$ implies $i=k$ and $j=\ell$.

Proof. Chips and wires are connected circuits. Suppose that $\mathfrak{p}$ is a non elementary circuit which does not satisfy the property of 3 : there exists $i \neq k$ and $j \neq \ell$ such that $n_{1}+\cdots+n_{i}=p_{1}+\cdots+p_{j}$. Then

$$
\mathfrak{p}=\begin{gathered}
\left(\mathfrak{p}_{1} \leftrightarrow \cdots \leftrightarrow \mathfrak{p}_{i}\right) \\
\uparrow \\
\left(\mathfrak{q}_{1} \leftrightarrow \cdots \leftrightarrow \mathfrak{q}_{j}\right)
\end{gathered} \leftrightarrow \begin{gathered}
\left(\mathfrak{p}_{i+1} \leftrightarrow \cdots \leftrightarrow \mathfrak{p}_{k}\right) \\
\left(\mathfrak{q}_{j+1} \leftrightarrow \cdots \leftrightarrow \mathfrak{q}_{\ell}\right)
\end{gathered}
$$

\subsection{Other free PROs}

The aim of this section is to discuss about how to represent the elements of a free PRO in the general case. Let $\mathcal{X}=\cup_{m, n \geq 0} \mathcal{X}_{m, n}$ be a bigraded set. In the previous section, we treat the case where $\mathcal{X}_{n, 0}=\mathcal{X}_{0, m}=\emptyset$ which seems to be one of the simplest cases. In general, we have more relations in $\mathcal{F}(X)$ which are consequences of the interchange law. Indeed, we have the following proposition. 
Proposition 3. Let $\mathcal{P}=\bigcup_{m, n} \mathcal{P}_{m, n}$ be a PRO. Let $\mathfrak{p} \in \mathcal{P}_{0, n}$ and $\mathfrak{q} \in \mathcal{P}_{m, 0}$. We have

$$
\mathfrak{p} \leftrightarrow \mathfrak{q}=\begin{aligned}
& \mathfrak{q} \\
& \mathfrak{l} \\
& \mathfrak{p}
\end{aligned}=\mathfrak{q} \leftrightarrow \mathfrak{p} .
$$

Moreover if $m=n=0$ then we have $\begin{aligned} & \mathfrak{p}= \\ & \mathfrak{q} \\ & \mathfrak{q}\end{aligned}$.

Proof. This comes from

$$
\begin{aligned}
\mathfrak{p} \leftrightarrow \mathfrak{q} & =\left(\begin{array}{l}
\oslash \\
\mathfrak{1} \\
\mathfrak{p}
\end{array}\right) \leftrightarrow\left(\begin{array}{l}
\mathfrak{q} \\
\mathfrak{l} \\
\oslash
\end{array}\right)=\begin{array}{c}
(\oslash \leftrightarrow \mathfrak{q}) \\
(\mathfrak{p} \leftrightarrow \oslash)
\end{array}=\begin{array}{c}
(\mathfrak{q} \leftrightarrow \oslash) \\
\mathfrak{\imath} \\
(\oslash \leftrightarrow \mathfrak{p})
\end{array} \\
& =\left(\begin{array}{l}
\mathfrak{q} \\
\mathfrak{1} \\
\oslash
\end{array}\right) \leftrightarrow\left(\begin{array}{l}
\oslash \\
\mathfrak{l} \\
\mathfrak{p}
\end{array}\right)=\mathfrak{q} \leftrightarrow \mathfrak{p}
\end{aligned}
$$

The special case $m=n=0$ is known as the Eckmann-Hilton argument2] [11]. Proposition[3implies that it is rather difficult to describe in a combinatorial way the element of a generic free Pro. For instance, the drawings $\mathrm{A}$ B $\mathrm{B}$ and $\mathrm{B}$ A must denote the same element which differs from the element whose drawing is

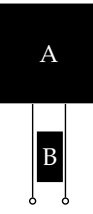

This suggests that one has to include in the description of the circuits a topological notion of zone. For instance, we can associate the element

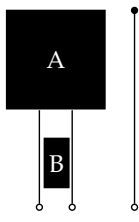

with the three zones below

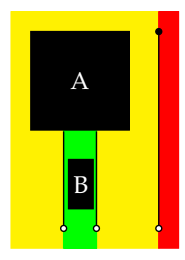

But, the description of the vertical composition in terms of zones seems to be complicated as suggested by the following example. Consider the element above with three zones together with the element represented by

\footnotetext{
${ }^{2}$ The Eckmann-Hilton argument states that if a set $X$ is endowed with two unital binary operations $\cdot$ and $\star$ satisfying $(a \star b) \cdot(c \star d)=(a \cdot c) \star(b \cdot d)$, then $\star$ and $\cdot$ are the same commutative and associative operation.
} 


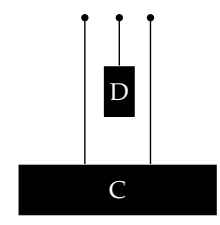

which has two zones. Their vertical composition has only one zone and admits the following graphical representations

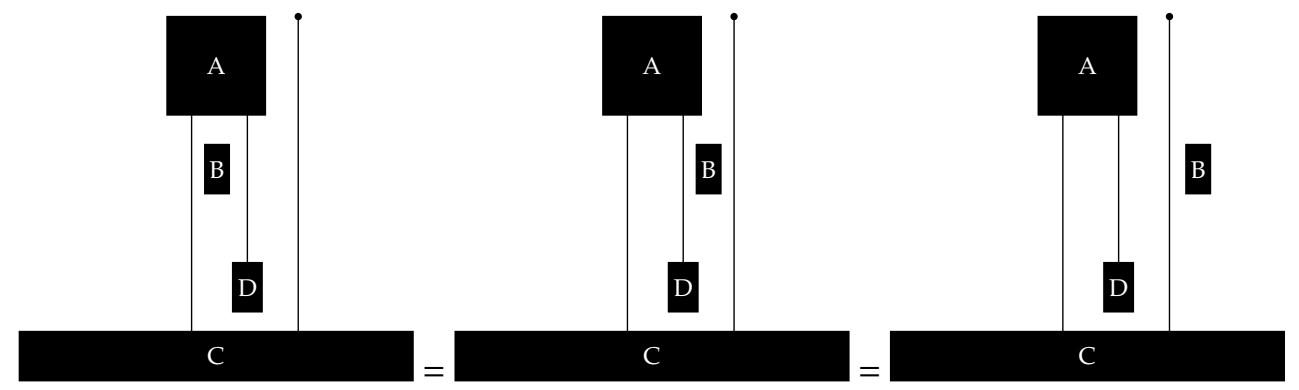

Notice that certain cases are simpler, as suggested by the following remark.

\subsection{Path colored PROs}

Let $C=\cup_{n, m \geq 1} C_{n, m}$ be a graded set of chips and $N>0$. We construct the set $\operatorname{Steps}(C, N)$ of the triplets $\left(\begin{array}{l}I \\ \mathfrak{c} \\ J\end{array}\right)$ with $\mathfrak{c} \in C$ and $I \in[N]^{n}$ and $J \in[N]^{m}$ when $\mathfrak{c} \in C_{n, m}$ (equivalently we label the inputs and the outputs of each chips by numbers in $[N])$. The set $\mathcal{P} a t h s(C, N)$ of the paths is obtained recursively as follows: a path is

- either the empty path denoted by $\oslash$ with 0 input and 0 output,

- or a labelled wire $\stackrel{i}{i}_{i}^{i}$ with 1 input and 1 output,

- or a labelled chips $\mathrm{c} \in \operatorname{Steps}(C, N)$,

- or the juxtaposition $\mathfrak{p p}^{\prime}$ of two paths with the rule $\oslash \mathfrak{p}=\mathfrak{p} \oslash=\mathfrak{p}$. The inputs (resp. the outputs) of $\mathfrak{p p}^{\prime}$ are obtained by catening the inputs (resp. the outputs) of $\mathfrak{p}$ and those of $\mathfrak{p}^{\prime}$. This operation will be denoted by $\leftrightarrow$.

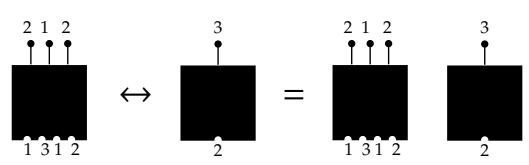

Figure 3: An example of horizontal composition of two paths.

- or the connexion of a $(I, J)$-path $p$ to a $(J, K)$-path $q$ obtained by connecting each input labelled by $i$ in $p$ to the output labelled by $i$ in $\mathfrak{q}$ by a wire labelled by $i$ with the rule: connecting a $(I, J)$-path to a list of labelled wires let the path unchanged. The outputs of the connections are the output of $p$ and the inputs are those of $q$. We denote by $\uparrow$ this operation. 


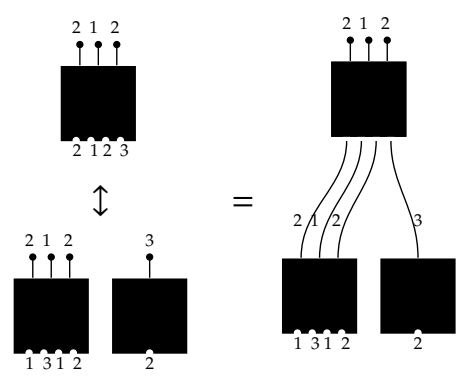

Figure 4: An example of vertical composition of two paths.

The set $\mathcal{P}$ aths $(C, N)$ is graded by the colors of the inputs and the outputs of the paths. For simplicity we denote by $\operatorname{In}(\mathfrak{q})$ (resp. Out(q)) the vector of the colors of the input (resp. output) of $q$. Straightforwardly from the definition:

Proposition 4. Paths $(C, N)$ is a colored PRO.

We define $u: \mathcal{P a t h s}(C, N) \longrightarrow \mathcal{F}(C)$ the unlabeling map that associate to each paths $\mathfrak{p}$ the circuit $u(\mathfrak{p})$ obtained by removing the labels from the inputs, the outputs and the connections of $\mathfrak{p}$ (see 5 for an example).

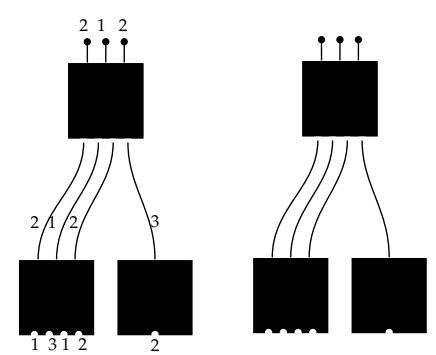

Figure 5: A path and its associated circuit.

The following result are easy to obtain:

\section{Lemma 2. 1. The sets}

$$
\left\{\mathfrak{q}_{1} \leftrightarrow \mathfrak{q}_{2}: u\left(\mathfrak{q}_{1}\right)=\mathfrak{p}_{1}, u\left(\mathfrak{q}_{2}\right)=\mathfrak{p}_{2}, \operatorname{In}\left(\mathfrak{q}_{1}\right)=I_{1}, \operatorname{In}\left(\mathfrak{q}_{2}\right)=I_{2}, \operatorname{Out}\left(\mathfrak{q}_{1}\right)=J_{1}, \text { and } \operatorname{Out}\left(\mathfrak{q}_{2}\right)=J_{2}\right\}
$$

and

$$
\left\{\mathfrak{q}: u(\mathfrak{q})=\mathfrak{p}_{1} \leftrightarrow \mathfrak{p}_{2}, \operatorname{Out}(\mathfrak{q})=I_{1} I_{2}, \text { and } \operatorname{In}(\mathfrak{q})=J_{1} J_{2}\right\}
$$

are equal.

2. The sets

$$
\left\{\begin{array}{c}
\mathfrak{q}^{\prime} \\
\mathfrak{i} \\
\mathfrak{q}^{\prime \prime}
\end{array}: u\left(\mathfrak{q}^{\prime}\right)=\mathfrak{p}^{\prime}, u\left(\mathfrak{q}^{\prime \prime}\right)=\mathfrak{p}^{\prime \prime}, \operatorname{In}\left(\mathfrak{q}^{\prime \prime}\right)=J, \operatorname{In}\left(\mathfrak{q}^{\prime}\right)=\operatorname{Out}\left(\mathfrak{q}^{\prime \prime}\right), \operatorname{Out}\left(\mathfrak{q}^{\prime}\right)=I\right\}
$$

and

$$
\left\{\mathfrak{q}: u(\mathfrak{q})=\begin{array}{c}
\mathfrak{p}^{\prime} \\
\mathfrak{l} \\
\mathfrak{p}^{\prime \prime}
\end{array}, \operatorname{Out}(\mathfrak{q})=I \text {, and } \operatorname{In}(\mathfrak{q})=J\right\}
$$


are equal.

\section{A PRO structure on hypermatrices}

\subsection{Hypermatrices}

An hypermatrix is a family of elements indexed by a list of integers $i_{1}, \ldots, i_{k}$ where $i_{j} \in\left[N_{j}\right]:=$ $\left\{1, \ldots, N_{j}\right\}$. The hypermatrices here are such that all the $N_{j}$ are the same. Furthermore we split the list of the indices into two parts in the aim to coincide with the structure of PRO.

Definition 2. Let $\mathbb{K}$ be a commutative semiring and $N, p, q \in \mathbb{N}, N>0$. We define

$$
\mathbb{K}(N, m, n):=\left\{\left(\begin{array}{l}
\mathrm{I} \\
\mathfrak{a} \\
j
\end{array}\right)_{\mathrm{I} \in[N]^{m}, J \in[N]^{n}}: \stackrel{\underset{\mathfrak{I}}{\mathfrak{a}} \in \mathbb{K}}{\mathrm{K}}\right\}
$$

with the special case $\mathbb{K}(N, 0,0):=\mathbb{K}$. We endow $\mathbb{K}(N):=\bigcup_{m, n} \mathcal{M}(N, m, n)$ with the three operators:

- We define $\leftrightarrow: \mathbb{K}(N, m, n) \times \mathbb{K}\left(N, m^{\prime}, n^{\prime}\right) \rightarrow \mathbb{K}\left(N, m+m^{\prime}, n+n^{\prime}\right)$ as follows. Consider $\mathbf{A}=\left(\begin{array}{l}\mathbb{I} \\ \mathfrak{a} \\ j\end{array}\right)_{\substack{\mathrm{I} \in\left[\mathbb{N}^{m} \\ j \in \mathbb{N}\right]^{n}}}$ and

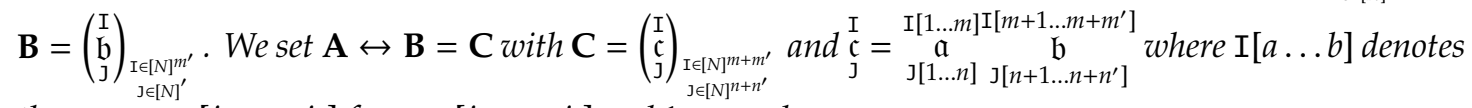
the sequence $\left[i_{a}, \ldots, i_{b}\right]$ for $\mathrm{I}=\left[i_{1}, \ldots, i_{s}\right]$ and $1 \leq a \leq b \leq s$.

- We define $\uparrow: \mathbb{K}(N, m, n) \times \mathbb{K}(N, n, p) \rightarrow \mathbb{K}(N, m, p)$ as follows. Consider $\mathbf{A}=\left(\begin{array}{l}\mathbb{I} \\ \mathfrak{a} \\ j\end{array}\right)_{\substack{\mathbb{I} \in\left[N m^{m} \\ j \in[\mathbb{N}]^{n}\right.}}$ and $\mathbf{B}=\left(\begin{array}{l}\mathbb{I} \\ \mathfrak{b} \\ J\end{array}\right)_{\substack{\mathbb{I} \in[N]^{n} \\ j \in[N]^{n}}}$.

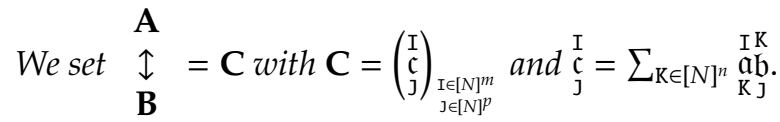

- Also each $\mathbb{K}(N, m, n)$ is naturally endowed with a structure of $\mathbb{K}$-module.

For the sake of simplicity, when computing, we write an hypermatrix of $\mathbb{K}(N, m, n)$ as a rectangular $N^{m} \times N^{n}$-matrix. With this notation, the operation $\uparrow$ is assimilated with the classical product of matrices and $\leftrightarrow$ with the Kronecker product.

Example 3. Let $\mathbf{A}=\left(\begin{array}{l}\mathrm{I} \\ \mathfrak{a} \\ J\end{array}\right)_{\mathrm{I} \in[2]^{2}, J \in[2]^{2}}$ and $\mathbf{B}=\left(\begin{array}{l}\mathrm{I} \\ \mathfrak{b} \\ \mathrm{J}\end{array}\right)_{\mathrm{I} \in[2]^{2}, J \in[2]^{2}}$. These matrices have the following 2dimensional representation

$$
\mathbf{A}=\left[\begin{array}{c|c|c|c}
11 & 11 & 11 & 11 \\
\mathfrak{a} & \mathfrak{a} & \mathfrak{a} & \mathfrak{a} \\
11 & 12 & 21 & 22 \\
\hline 12 & 12 & 12 & 12 \\
\mathfrak{a} & \mathfrak{a} & \mathfrak{a} & \mathfrak{a} \\
11 & 12 & 21 & 22 \\
\hline 21 & 21 & 21 & 21 \\
\mathfrak{a} & \mathfrak{a} & \mathfrak{a} & \mathfrak{a} \\
11 & 12 & 21 & 22 \\
\hline 22 & 22 & 22 & 22 \\
\mathfrak{a} & \mathfrak{a} & \mathfrak{a} & \mathfrak{a} \\
11 & 12 & 21 & 22
\end{array}\right] \text { and } \mathbf{B}=\left[\begin{array}{c|c|c|c}
11 & 11 & 11 & 11 \\
\mathfrak{b} & \mathfrak{b} & \mathfrak{b} & \mathfrak{b} \\
11 & 12 & 21 & 22 \\
\hline 12 & 12 & 12 & 12 \\
\mathfrak{b} & \mathfrak{b} & \mathfrak{b} & \mathbf{b} \\
11 & 12 & 21 & 22 \\
\hline 21 & 21 & 21 & 21 \\
\mathfrak{b} & \mathfrak{b} & \mathfrak{b} & \mathfrak{b} \\
11 & 12 & 21 & 22 \\
\hline 22 & 22 & 22 & 22 \\
\mathfrak{b} & \mathfrak{b} & \mathfrak{b} & \mathbf{b} \\
11 & 12 & 21 & 22
\end{array}\right] .
$$

We have

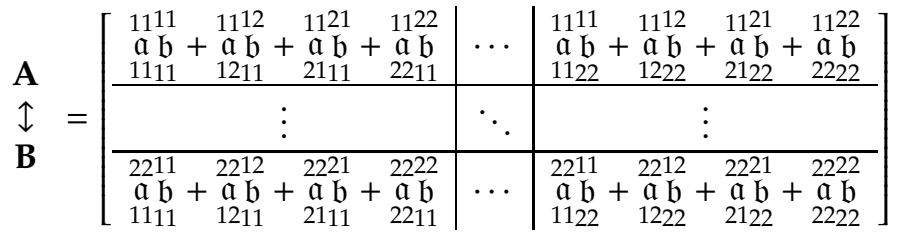


and

\begin{tabular}{|c|c|c|c|c|c|c|c|c|c|}
\hline & 1111 & 11 & & 1111 & & 1111 & 1111 & 1111 & \\
\hline & $\begin{array}{l}a b \\
1111\end{array}$ & $\begin{array}{l}\mathfrak{a} b \\
1112\end{array}$ & $\begin{array}{l}a b b \\
1121\end{array}$ & $\begin{array}{l}a b \\
1122 \\
120\end{array}$ & $\cdots$ & $\begin{array}{l}a, b \\
2211\end{array}$ & $\begin{array}{l}a b \\
2212\end{array}$ & $\begin{array}{l}a b \\
2221\end{array}$ & $\begin{array}{l}a b b \\
2222\end{array}$ \\
\hline & 1112 & 1112 & 1112 & 1112 & & 1112 & 1112 & 1112 & 1112 \\
\hline & ${ }_{111}^{\mathfrak{a}} \mathfrak{b}$ & $\mathfrak{a} \mathfrak{b}$ & $\mathfrak{a} \mathfrak{b}$ & $\mathfrak{a} b$ & $\cdots$ & $\mathfrak{a} \mathfrak{b}$ & $\mathfrak{a} b$ & $\mathfrak{a} b$ & $a b$ \\
\hline & $\frac{1111}{1121}$ & $\frac{1121}{1121}$ & $\frac{1121}{1121}$ & $\frac{1124}{1121}$ & & $\frac{211}{1121}$ & $\frac{2121}{1121}$ & $\frac{2<1}{1121}$ & \\
\hline & $\begin{array}{l}a \mathfrak{b} \\
1111\end{array}$ & $\begin{array}{l}\mathfrak{a} \mathfrak{b} \\
1111\end{array}$ & $\begin{array}{c}a b \\
112\end{array}$ & $\begin{array}{l}a \mathfrak{a} \mathfrak{b} \\
1112\end{array}$ & $\cdots$ & $\begin{array}{c}a, b \\
2211\end{array}$ & $\begin{array}{l}\mathfrak{a} 2 \mathfrak{b} \\
2212\end{array}$ & $\begin{array}{l}a \mathfrak{a} b \\
2221\end{array}$ & $\begin{array}{c}\mathfrak{a} b \mathfrak{b} \\
2222\end{array}$ \\
\hline & 1122 & 1122 & 1122 & 1122 & & 1122 & 1122 & 1122 & 1122 \\
\hline & $\begin{array}{l}\mathfrak{a} b \mathbf{b} \\
1111\end{array}$ & $\begin{array}{l}a b \\
1112\end{array}$ & $\begin{array}{l}a b \\
1121\end{array}$ & $\begin{array}{l}a b \\
1122\end{array}$ & $\cdots$ & $\begin{array}{r}a, b \\
2211\end{array}$ & $\begin{array}{c}a b \\
2212\end{array}$ & $\begin{array}{c}a b \\
2221\end{array}$ & ${ }_{222}^{a} b$ \\
\hline \multirow{10}{*}{$\mathbf{A} \leftrightarrow \mathbf{B}=$} & & & & & $\ddots$ & & & & \\
\hline & 2211 & 2212 & 2221 & 2222 & & 2211 & 2212 & & \\
\hline & $\mathfrak{a} \mathfrak{b}$ & $\mathfrak{a} b$ & $\mathfrak{a}$ & $\mathfrak{a} \mathfrak{b}$ & $\cdots$ & $a b$ & $\mathfrak{a}$ & $\mathfrak{a} \mathbf{b}$ & $\mathfrak{a} \mathfrak{b}$ \\
\hline & $\frac{1111}{2211}$ & & & & & & & $\frac{2411}{2221}$ & \\
\hline & $\mathfrak{a} \mathfrak{b}$ & $\mathfrak{a} b$ & $\mathfrak{a} \mathfrak{b}$ & $\mathfrak{a} \mathfrak{b}$ & & $a b$ & $\mathfrak{a b}$ & $a \mathfrak{b}$ & $a \mathfrak{a}$ \\
\hline & 1112 & 1112 & 1112 & 1112 & & 2212 & $\frac{2212}{2012}$ & $\frac{2212}{202}$ & \\
\hline & 2211 & 2212 & 2221 & 2222 & $\ldots$ & 2211 & 2212 & 2241 & 2242 \\
\hline & 1121 & 1121 & 1121 & $\begin{array}{l}1120 \\
1121\end{array}$ & & 2221 & 2221 & 2221 & \\
\hline & & 2212 & 2221 & 2222 & & & 2212 & 2221 & \\
\hline & $\begin{array}{l}a \mathfrak{a} \\
1122\end{array}$ & $\begin{array}{l}a b \\
1122\end{array}$ & $\begin{array}{l}\mathrm{a} b \mathrm{~b} \\
1122\end{array}$ & $\begin{array}{l}a b \\
1122\end{array}$ & & $\begin{array}{l}a \mathrm{~b} \\
2222\end{array}$ & $\begin{array}{l}a b \\
2222\end{array}$ & $\begin{array}{l}a b b \\
2222\end{array}$ & $\begin{array}{l}\mathrm{a} b \\
2222\end{array}$ \\
\hline
\end{tabular}

Proposition 5. $\mathbb{K}(N)$ is a $\mathbb{K}-$ ModPro.

Proof. It is easy to check that $\mathbb{K}(N)$ is a PRO where the graded $(1,1)$-vertical unity, $\mathbf{I}_{1}(N)$, is the $N \times N$ identity matrix and the horizontal unity is $\mathbf{I}_{0}(N)=1$. The left, right, up and down distributivity follows from the bilinearity of the product and Kronecker product of matrices.

We introduce for $K \in[N]^{p}, L \in[N]^{q}$ the hypermatrices $E(N, p, q ; K, L)=\left(\delta_{I K} \delta_{J L}\right)_{I \in[N]^{p}, \in[N]^{q}}$ where $\delta_{I K}=0$ if $I \neq K$ and 1 otherwise is the Kronecker delta.. As in the case of matrices, these elements play the role of basic elements allowing to decompose the hypermatrices.

Claim 1. For each $\mathbf{A}=\left(\begin{array}{l}I \\ \mathfrak{a} \\ J\end{array}\right)$, we have

$$
\mathbf{A}=\underset{+, J}{\mathfrak{a}} \stackrel{I}{\mathfrak{a}} \mathrm{E}(N, p, q ; I, J) .
$$

Furthermore this decomposition is unique.

Furthermore, we have for any $I \in[N]^{p}, J \in[N]^{q}, I^{\prime} \in[N]^{p^{\prime}}, J^{\prime} \in[N]^{q^{\prime}}$

$$
\mathbf{E}(N, p, q ; I, J) \leftrightarrow \mathbf{E}\left(N, p^{\prime}, q^{\prime} ; I^{\prime}, J^{\prime}\right)=\mathbf{E}\left(N, p+p^{\prime}, q+q^{\prime} ; I I^{\prime}, J J^{\prime}\right)
$$

where $\left[i_{1}, \ldots, i_{k}\right]\left[j_{1}, \ldots, j_{\ell}\right]=\left[i_{1}, \ldots, i_{k}, j_{1}, \ldots, j_{\ell}\right]$ denotes the catenation of the sequences, and for any $I \in[N]^{p}, J, J^{\prime} \in[N]^{q}$, and $K \in\left[N^{r}\right]$,

$$
\begin{gathered}
\mathbf{E}(N, p, q ; I, J) \\
\mathfrak{\imath} \\
\mathbf{E}\left(N, p, r ; J^{\prime}, K\right)
\end{gathered}=\delta_{J, J^{\prime}} \mathbf{E}(N, p, r ; I, K),
$$

where $\delta_{J J^{\prime}}=1$ if $J=J^{\prime}$ and 0 otherwise.

\subsection{Kronecker product}

If $I=\left[i_{1}, \ldots, i_{p}\right] \in(\mathbb{N}-\{0\})^{p}$ we will write $I \% N=\left[r_{1}, \ldots, r_{p}\right]$ and $I / N=\left[q_{1}, \ldots, q_{p}\right]$ where $i_{k}=\left(q_{k}-1\right) N+r_{k}$ and $1 \leq r_{k-1} \leq N$. 
The Kronecker product $\mathbf{A} \odot \mathbf{B}$ of two hypermatrices $\mathbf{A}=\left(\begin{array}{l}I \\ \mathfrak{a} \\ J\end{array}\right) \in \mathbb{K}(M, p, q), \mathbf{B}=\left(\begin{array}{l}I \\ \mathfrak{b} \\ J\end{array}\right) \in \mathbb{K}(N, p, q)$ is the hypermatrix $\mathbf{C}=\left(\begin{array}{l}I \\ \mathfrak{c} \\ J\end{array}\right) \in \mathbb{K}(M N, p, q)$ defined by

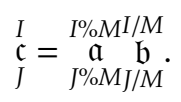

For instance we have the following identity

$$
\begin{aligned}
& \mathbf{E}\left(M, p, q ;\left[r_{1}, \ldots, r_{p}\right],\left[s_{1}, \ldots, s_{q}\right]\right) \odot \mathbf{E}\left(N, p, q ;\left[k_{1}, \ldots, k_{p}\right],\left[\ell_{1}, \ldots, \ell_{q}\right]\right)= \\
& \mathbf{E}\left(M N, p, q ;\left[\left(k_{1}-1\right) M+r_{1}, \ldots,\left(k_{p}-1\right) M+r_{p}\right],\left[\left(\ell_{1}-1\right) M+s_{1}, \ldots,\left(\ell_{q}-1\right) M+s_{q}\right)\right] .
\end{aligned}
$$

Or equivalently,

$$
\mathbf{E}(M N, p, q ; I, J)=\mathbf{E}(M, p, q ; I \% M, J \% M) \odot \mathbf{E}(N, p, q ; I / N, J / N)
$$

with the same notation as in Equation (17).

Let us examine the first properties of this construction.

Lemma 3. We have $(\mathbf{A}+\mathbf{B}) \odot \mathbf{C}=(\mathbf{A} \odot \mathbf{C})+(\mathbf{B} \odot \mathbf{C})$ and $\mathbf{A} \odot(\mathbf{B}+\mathbf{C})=(\mathbf{A} \odot \mathbf{B})+(\mathbf{A} \odot \mathbf{C})$.

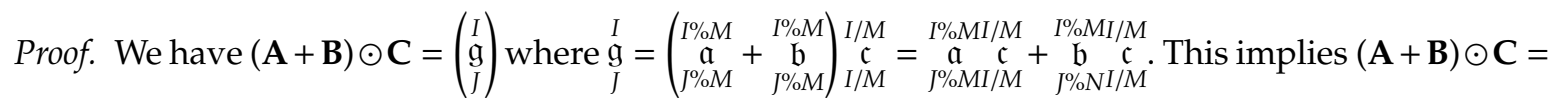
$(\mathbf{A} \odot \mathbf{C})+(\mathbf{B} \odot \mathbf{C})$. The second identities is proved in a similar way.

Lemma 4. We have $\mathrm{r} .(\mathbf{A} \odot \mathbf{B})=(\mathfrak{r} . \mathbf{A}) \odot \mathbf{B}=\mathbf{A} \odot(\mathfrak{r} . \mathbf{B})$

Proof. This is straightforward from the definition.

The Kronecker product allows to give an analogue to a result which is classical for matrices.

Lemma 5. The $\mathbb{K}$-module $\mathbb{K}(M N ; p, q)$ and $\mathbb{K}(M ; p, q) \otimes \mathbb{K}(N ; p, q)$ are isomorphic. An explicit morphism $\phi$ sends each $\mathbf{A} \otimes \mathbf{B}$ to $\mathbf{A} \odot \mathbf{B}$.

Proof. The fact that $\phi$ is an isomorphism comes that one can construct explicitly its inverse as the unique morphism satisfying $\phi^{-1}(\mathbf{E}(M N, p, q ; I, J))=\phi^{-1}(\mathbf{E}(M, p, q ; I \% M, J \% M) \odot \mathbf{E}(M, p, q ; I / M, J / M))=$ $\mathbf{E}(M, p, q ; I \% M, J \% M) \otimes \mathbf{E}(M, p, q ; I / M, J / M)$.

The set $\mathbb{K}(M) \otimes \mathbb{K}(N):=\bigcup_{p, q} \mathbb{K}(M ; p, q) \otimes \mathbb{K}(N ; p, q)$ is naturally endowed with a structure of Pro. It suffices to set $\left(\mathbf{A} \otimes \mathbf{A}^{\prime}\right) \leftrightarrow\left(\mathbf{B} \otimes \mathbf{B}^{\prime}\right)=(\mathbf{A} \leftrightarrow \mathbf{B}) \otimes\left(\mathbf{A}^{\prime} \leftrightarrow \mathbf{B}^{\prime}\right)$ and $\begin{gathered}\left(\mathbf{A} \otimes \mathbf{A}^{\prime}\right) \\ \begin{array}{c}\uparrow \\ \left(\mathbf{B} \otimes \mathbf{B}^{\prime}\right)\end{array}\end{gathered}=\begin{aligned} & \mathbf{A} \\ & \begin{array}{l}\uparrow \\ \mathbf{B}\end{array}\end{aligned} \begin{gathered}\mathbf{A}^{\prime} \\ \mathbf{B}^{\prime}\end{gathered}$. Furthermore, one checks that the action of $\mathbb{K}$ on each $\mathbb{K}(M ; p, q) \otimes \mathbb{K}(N ; p, q)$ is compatible with the operations $\leftrightarrow$ and $\downarrow$ in the sense of the definition of ModPro. So, $\mathbb{K}(M) \otimes \mathbb{K}(N)$ is a $\mathbb{K}$-ModPro.

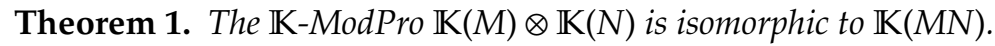

Proof. From Lemma 5 it suffices to prove that $\mathbb{K}(M) \otimes \mathbb{K}(N)$ and $\mathbb{K}(N N)$ are isomorphic as PROs. Consider, as in Lemma 5, the map $\phi$ sending each $(\mathbf{A} \otimes \mathbf{B})$ to $\mathbf{A} \odot \mathbf{B}$. We have to prove that it is a morphism of PRO.

- Image of the units

Obviously $\phi\left(\mathbf{I}(M)^{\leftrightarrow p} \otimes \mathbf{I}(N)^{\leftrightarrow p}\right)=\mathbf{I}(M)^{\leftrightarrow p} \odot \mathbf{I}(N)^{\leftrightarrow p}=\mathbf{I}(M N)^{\leftrightarrow p}$ and $\phi\left(\mathbf{I}_{0}(M) \otimes \mathbf{I}_{0}(N)\right)=0=$ $\mathbf{I}_{0}(M N)$. 
- Compatibility with $\leftrightarrow$

Let $\mathbf{A}=\left(\begin{array}{c}I_{1} \% N \\ \mathfrak{a} \\ J_{1} \% N\end{array}\right) \in \mathbb{K}\left(M ; p_{A}, q_{A}\right), \mathbf{B}=\left(\begin{array}{c}I_{1} \% N \\ \mathfrak{b} \\ J_{1} \% N\end{array}\right) \in \mathbf{K}\left(M ; p_{B}, q_{B}\right), \mathbf{A}^{\prime}=\left(\begin{array}{c}I_{1} \% N \\ \mathfrak{a}^{\prime} \\ J_{1} \% N\end{array}\right) \in \mathbb{K}\left(N ; p_{A}, q_{A}\right)$ and $\mathbf{B}^{\prime}=$ $\left(\begin{array}{c}I_{1} \% N \\ \mathfrak{b}^{\prime} \\ J_{1} \% N\end{array}\right) \in \mathbb{K}\left(N ; p_{B}, q_{B}\right)$. We have

$$
\phi\left((\mathbf{A} \otimes \mathbf{B}) \leftrightarrow\left(\mathbf{A}^{\prime} \otimes \mathbf{B}^{\prime}\right)\right)=\phi\left((\mathbf{A} \leftrightarrow \mathbf{B}) \otimes\left(\mathbf{A}^{\prime} \leftrightarrow \mathbf{B}^{\prime}\right)\right)=\left(\begin{array}{c}
I \\
\mathfrak{c} \\
J
\end{array}\right)_{\substack{I \in\left[N N^{\prime} p^{p} A_{A}+p_{B} \\
j \in\left[N N^{\prime}\right]^{q} A^{+} q_{B}\right.}}
$$

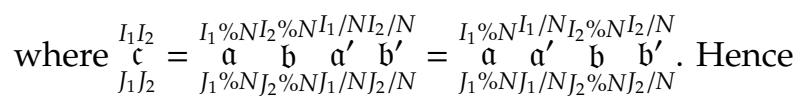

$$
\phi\left((\mathbf{A} \otimes \mathbf{B}) \leftrightarrow\left(\mathbf{A}^{\prime} \otimes \mathbf{B}^{\prime}\right)\right)=\left(\phi(\mathbf{A}) \odot \phi\left(\mathbf{B}^{\prime}\right)\right) \leftrightarrow\left(\phi(\mathbf{B}) \odot \phi\left(\mathbf{B}^{\prime}\right)\right)=\left(\phi\left(\mathbf{A} \otimes \mathbf{A}^{\prime}\right) \leftrightarrow \phi\left(\mathbf{B} \otimes \mathbf{B}^{\prime}\right)\right)
$$

- Compatibility with $\uparrow$

Let $\mathbf{A}=\left(\begin{array}{c}I_{1} \% N \\ \mathfrak{a} \\ J_{1} \% N\end{array}\right) \in \mathbb{K}(M ; p, q), \mathbf{B}=\left(\begin{array}{c}I_{1} \% N \\ b \\ b N \\ J_{1} \% N\end{array}\right) \in \mathbf{K}(M ; q, r), \mathbf{A}^{\prime}=\left(\begin{array}{c}I_{1} \% N \\ \mathfrak{a}^{\prime} \\ J_{1} \% N\end{array}\right) \in \mathbb{K}(N ; p, q)$ and $\mathbf{B}^{\prime}=\left(\begin{array}{c}I_{1} \% N \\ \mathfrak{b}^{\prime} \\ J_{1} \% N\end{array}\right) \in$ $\mathbb{K}(N ; q, r)$. We have

$$
\phi\left(\begin{array}{c}
\mathbf{A} \otimes \mathbf{A}^{\prime} \\
\mathfrak{I} \\
\mathbf{B} \otimes \mathbf{B}^{\prime}
\end{array}\right)=\left(\begin{array}{c}
\mathbf{A} \\
\mathfrak{I} \\
\mathbf{B}
\end{array}\right) \odot\left(\begin{array}{c}
\mathbf{A}^{\prime} \\
\mathfrak{I} \\
\mathbf{B}^{\prime}
\end{array}\right)=\left(\begin{array}{l}
I \\
\mathfrak{c} \\
J
\end{array}\right)
$$

where

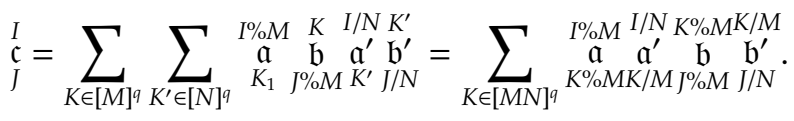

Hence we recognize:

$$
\phi\left(\begin{array}{c}
\mathbf{A}_{1} \otimes \mathbf{B}_{1} \\
\hat{\imath} \\
\mathbf{A}_{2} \otimes \mathbf{B}_{2}
\end{array}\right)=\begin{gathered}
\phi\left(\mathbf{A}_{1} \otimes \mathbf{B}_{1}\right) \\
\uparrow \\
\phi\left(\mathbf{A}_{2} \otimes \mathbf{B}_{2}\right)
\end{gathered} .
$$

This proves the result. that

The proof of Theorem 1 exhibits an explicit isomorphism sending each $\mathbf{A} \otimes \mathbf{B}$ to $\mathbf{A} \odot \mathbf{B}$. It follows

$$
(\mathbf{A} \leftrightarrow \mathbf{B}) \odot\left(\mathbf{A}^{\prime} \leftrightarrow \mathbf{B}^{\prime}\right)=\left(\mathbf{A} \odot \mathbf{A}^{\prime}\right) \leftrightarrow\left(\mathbf{B} \odot \mathbf{B}^{\prime}\right) .
$$

Indeed, the preimage by $\phi$ of this identity is

$$
(\mathbf{A} \leftrightarrow \mathbf{B}) \otimes\left(\mathbf{A}^{\prime} \leftrightarrow \mathbf{B}^{\prime}\right)=\left(\mathbf{A} \otimes \mathbf{A}^{\prime}\right) \leftrightarrow\left(\mathbf{B} \otimes \mathbf{B}^{\prime}\right)
$$

In the same way, we obtain

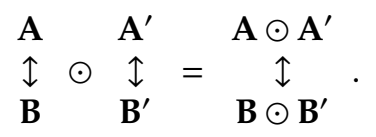

\subsection{Quasi-direct sum}

Let $\mathbf{A}=\left(\begin{array}{l}I \\ \mathfrak{a} \\ J\end{array}\right) \in \mathbb{K}(M ; p, q)$ and $\mathbf{B}=\left(\begin{array}{l}I \\ \mathfrak{b} \\ J\end{array}\right) \in \mathbb{K}(N, p, q)$. We define the quasi-direct sum of $\mathbf{A}$ and $\mathbf{B}$ by

$$
\mathbf{A} \hat{\oplus} \mathbf{B}:=\left(\begin{array}{l}
I \\
c \\
J
\end{array}\right)_{I, J} \in \mathbb{K}(M+N, p, q)
$$


with

$$
\underset{\mathfrak{c}}{I}= \begin{cases}I & \text { if } I \in[M]^{p} \text { and } J \in[M]^{q}, \\ J & \\ I-[M, \ldots, M] & \text { if } I-[M, \ldots, M] \in[N]^{p} \text { and } J-[M, \ldots, M] \in[N]^{q}, \\ J-[M, \ldots, M] & \text { otherwise. }\end{cases}
$$

From this definition, one has

$$
\mathbf{I}(M) \hat{\oplus} \mathbf{I}(N)=\mathbf{I}(M+N) .
$$

and for any $I \in[M]^{p}, J \in[M]^{q}, I^{\prime}=\left[i_{1}^{\prime}, \ldots, i_{p}^{\prime}\right] \in[N]^{p}$, and $J^{\prime}=\left[j_{1}^{\prime}, \ldots, j_{q}^{\prime}\right] \in[N]^{q}$, we have

$$
\mathbf{E}(M, p, q ; I, J) \hat{\oplus} \mathbf{E}\left(N, p, q ; I^{\prime}, J^{\prime}\right)=\mathbf{E}(M+N, p, q ; I, J)+\mathbf{E}\left(M+N, p, q ;\left[i_{1}^{\prime}, \ldots, i_{p}^{\prime}\right]+\left[M^{p}\right],\left[j_{1}^{\prime}, \ldots, j_{q}^{\prime}\right]+\left[M^{q}\right]\right),
$$

where $\left[M^{n}\right]=[\overbrace{M, \ldots, M}^{n \times}]$. We do not call this operation "direct sum" because it not compatible with the horizontal composition as shown by the simplest counter example below.

Consider the hypermatrix $(\mathbf{I}(1) \hat{\oplus} \mathbf{I}(1)) \leftrightarrow(\mathbf{I}(1) \hat{\oplus} \mathbf{I}(1))=\mathbf{I}(2)^{\leftrightarrow 2}$ which differs from $(\mathbf{I}(1) \leftrightarrow \mathbf{I}(1)) \hat{\oplus}(\mathbf{I}(1) \leftrightarrow \mathbf{I}(1))$. Indeed,

$$
(\mathbf{I}(1) \leftrightarrow \mathbf{I}(1)) \hat{\oplus}(\mathbf{I}(1) \leftrightarrow I(1))=\mathbf{I}(1)^{\leftrightarrow 2} \hat{\oplus} \mathbf{I}(1)^{\leftrightarrow 2}=\left(\begin{array}{l}
I \\
l \\
J
\end{array}\right)_{I, J}
$$

where

$$
\stackrel{I}{I}= \begin{cases}1 & \text { if } I=J \text { and }(I=[1,1] \text { or } I=[2,2]), \\ 0 & \text { otherwise. }\end{cases}
$$

We remark that $\underset{12}{12}=0$ whilst the corresponding entry in $\mathbf{I}(2)^{\leftrightarrow 2}$ equals 1 .

So in general

$$
\left(\mathbf{A} \hat{\oplus} \mathbf{A}^{\prime}\right) \leftrightarrow\left(\mathbf{B} \hat{\oplus} \mathbf{B}^{\prime}\right) \neq(\mathbf{A} \leftrightarrow \mathbf{B}) \hat{\oplus}\left(\mathbf{A}^{\prime} \leftrightarrow \mathbf{B}^{\prime}\right)
$$

However the quasi-direct sum is compatible with the vertical composition.

Proposition 6. Let $\mathbf{A} \in \mathbb{K}(M ; p, q), \mathbf{B} \in \mathbb{K}(M ; q, r), \mathbf{A}^{\prime} \in \mathbb{K}(N, p, q)$ and $\mathbf{B}^{\prime} \in \mathbb{K}(N, q, r)$ be four hypermatrices. We have

$$
\underset{\mathbf{A} \hat{\oplus} \mathbf{A}^{\prime}}{\stackrel{1}{\mathbf{\oplus} \mathbf{B}^{\prime}}}=\left(\begin{array}{c}
\mathbf{A} \\
\uparrow \\
\mathbf{B}
\end{array}\right) \hat{\oplus}\left(\begin{array}{c}
\mathbf{A}^{\prime} \\
\uparrow \\
\mathbf{B}^{\prime}
\end{array}\right)
$$

Proof. For convenience let us set $\mathbf{A} \hat{\oplus} \mathbf{A}^{\prime}=\left(\begin{array}{l}I \\ \mathfrak{c} \\ I\end{array}\right)_{I J}, \mathbf{B} \hat{\oplus} \mathbf{B}^{\prime}=\left(\begin{array}{l}I \\ \mathfrak{d} \\ J\end{array}\right)_{I J}, \begin{gathered}\mathbf{A} \hat{\oplus} \mathbf{A}^{\prime} \\ \hat{\mathbf{D}} \\ \mathbf{B} \hat{\oplus} \mathbf{B}^{\prime}\end{gathered}=\left(\begin{array}{l}I \\ \mathfrak{e} \\ I\end{array}\right)_{I J}$, and $\left(\begin{array}{c}\mathbf{A} \\ \hat{\downarrow} \\ \mathbf{B}\end{array}\right) \hat{\oplus}\left(\begin{array}{c}\mathbf{A}^{\prime} \\ \mathfrak{I} \\ \mathbf{B}^{\prime}\end{array}\right)=$ $\left(\begin{array}{l}I \\ g \\ J\end{array}\right)_{I J}$. Our goal consists to prove that for each $I, J, \underset{J}{\stackrel{I}{e}}=\underset{J}{\stackrel{I}{g}}$. To this aim we write the entries of each

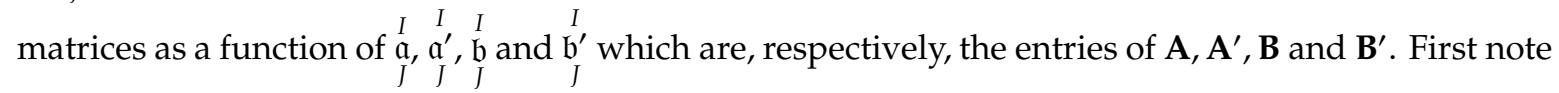
that

$$
\stackrel{I}{\mathfrak{e}}=\sum_{J \in[M+N]^{q}} \underset{\substack{I K \\
\mathcal{C D}}}{I K}=\left(\sum_{K \in[M]^{9}} \begin{array}{c}
I K \\
\mathfrak{C D} \\
K J
\end{array}\right)+\left(\sum_{K-[M, \ldots, M] \in[N]^{q}} \begin{array}{r}
I K J \\
\mathfrak{C D}
\end{array}\right)
$$

But the two sums in the right hand side can not be simultaneously non zero. Hence

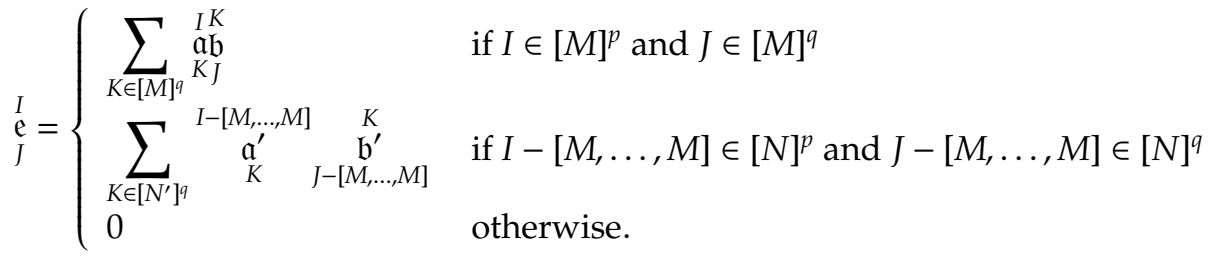


This is exactly the value of $\stackrel{I}{\mathfrak{g}}$.

Remark also that $\hat{\oplus}$ is bilinear

$$
\left(\mathbf{A}+\mathbf{A}^{\prime}\right) \hat{\oplus}\left(\mathbf{B}+\mathbf{B}^{\prime}\right)=\mathbf{A} \hat{\oplus} \mathbf{B}+\mathbf{A} \hat{\oplus} \mathbf{B}^{\prime}+\mathbf{A}^{\prime} \hat{\oplus} \mathbf{B}+\mathbf{A}^{\prime} \hat{\oplus} \mathbf{B}^{\prime}
$$

Consider now more complicated identities.

Lemma 6. Let $m_{a}, n_{a}, m_{b}, m_{c}, p_{q}, q_{d}, q_{e} \geq 0$ and $N_{0}, N_{1}, n_{b}, n_{c}, p_{e}>0$ such that $n_{a}+n_{b}>p_{d}$ and $n_{a}+n_{b}+n_{c}=$ $p_{q}+p_{e}$

- Let $\mathbf{A}=\left(\begin{array}{c}I \\ \mathfrak{a} \\ J\end{array}\right) \in \mathbb{K}\left(N_{0}+N_{1} ; m_{a}, n_{a}\right), \mathbf{D}=\left(\begin{array}{c}I \\ \mathfrak{d} \\ J\end{array}\right) \in \mathbb{K}\left(N_{0}+N_{1} ; p_{d}, q_{d}\right), \mathbf{B}_{i}=\left(\begin{array}{c}I \\ \mathfrak{b}_{i} \\ J\end{array}\right) \in \mathbb{K}\left(N_{i} ; m_{b}, n_{b}\right), \mathbf{C}_{i}=\left(\begin{array}{c}I \\ \mathfrak{c}_{i} \\ J\end{array}\right) \in$ $\mathbb{K}\left(N_{i} ; m_{c}, n_{c}\right)$ and $\mathbf{E}_{i}=\left(\begin{array}{c}I \\ e_{i} \\ J\end{array}\right) \in \mathbb{K}\left(N_{i} ; p_{e}, q_{e}\right)(i=0,1)$. One has

$$
\begin{gathered}
\mathbf{A} \leftrightarrow\left(\left(\mathbf{B}_{0} \leftrightarrow \mathbf{C}_{0}\right) \hat{\oplus}\left(\mathbf{B}_{1} \leftrightarrow \mathbf{C}_{1}\right)\right) \\
\mathbf{D} \leftrightarrow\left(\mathbf{E}_{0} \hat{\oplus} \mathbf{E}_{1}\right)
\end{gathered} \quad \begin{gathered}
\mathbf{A} \leftrightarrow\left(\mathbf{B}_{0} \hat{\oplus} \mathbf{B}_{1}\right) \leftrightarrow\left(\mathbf{C}_{0} \hat{\oplus} \mathbf{C}_{1}\right) \\
\hat{\downarrow} \\
\mathbf{D} \leftrightarrow\left(\mathbf{E}_{0} \hat{\oplus} \mathbf{E}_{1}\right)
\end{gathered}
$$

- Let $\mathbf{A}=\left(\begin{array}{l}I \\ \mathfrak{a} \\ J\end{array}\right) \in \mathbb{K}\left(N_{0}+N_{1} ; n_{a}, m_{a}\right), \mathbf{D}=\left(\begin{array}{c}I \\ \mathfrak{d} \\ J\end{array}\right) \in \mathbb{K}\left(N_{0}+N_{1} ; q_{d}, p_{d}\right), \mathbf{B}_{i}=\left(\begin{array}{c}I \\ \mathfrak{b}_{i} \\ J\end{array}\right) \in \mathbb{K}\left(N_{i} ; n_{b}, m_{b}\right), \mathbf{C}_{i}=\left(\begin{array}{c}I \\ \mathfrak{c}_{i} \\ J\end{array}\right) \in$ $\mathbb{K}\left(N_{i} ; n_{c}, m_{c}\right)$ and $\mathbf{E}_{i}=\left(\begin{array}{c}I \\ \mathfrak{e}_{i} \\ J\end{array}\right) \in \mathbb{K}\left(N_{i} ; q_{e}, p_{e}\right)(i=0,1)$. One has

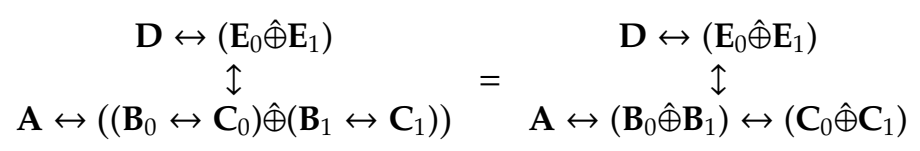

Proof. We prove only (27); the other identity being hence obtained by symmetry. From the bilinearity of $\hat{\oplus}, \leftrightarrow$ and $\uparrow$ and Claim 1 , it suffices to prove the result in the cases where $\mathbf{A}=\mathbf{E}\left(N_{0}+N_{1}, m_{a}, n_{a} ; I_{a}, J_{a}\right), \mathbf{D}=$ $\mathbf{E}\left(N_{0}+N_{1}, p_{d}, q_{d} ; I_{d}, J_{d}\right), \mathbf{B}_{i}=\mathbf{E}\left(N_{i}, m_{b}, n_{b} ; I_{b}^{(i)}, J_{b}^{(i)}\right), \mathbf{C}_{i}=\mathbf{E}\left(N_{i}, m_{c}, n_{c} ; I_{c}^{(i)}, J_{c}^{(i)}\right)$, and $\mathbf{E}_{i}=\mathbf{E}\left(N_{i}, p_{e}, q_{e} ; I_{e}^{(i)}, J_{e}^{(i)}\right)$. One has

$$
\begin{aligned}
& \mathbf{A} \leftrightarrow\left(\left(\mathbf{B}_{0} \leftrightarrow \mathbf{C}_{0}\right) \hat{\oplus}\left(\mathbf{B}_{1} \leftrightarrow \mathbf{C}_{1}\right)\right)=\mathbf{E}\left(N_{0}+N_{1}, m_{a}+m_{b}+m_{c}, n_{a}+n_{b}+n_{c} ; I_{a} I_{b}^{(0)} I_{c}^{(0)}, J_{a} J_{b}^{(0)} J_{c}^{(0)}\right) \\
& +\mathbf{E}\left(N_{0}+N_{1}, m_{a}+m_{b}+m_{c}, n_{a}+n_{b}+n_{c} ; I_{a} I_{b}^{(1)} I_{c}^{(1)}+\left[0^{m_{a}} N_{0}^{m_{b}+m_{v}}\right], J_{a} J_{b}^{(1)} J_{c}^{(1)}+\left[0^{n_{a}} N_{0}^{n_{b}+n_{v}}\right]\right)
\end{aligned}
$$

and

$$
\begin{aligned}
& \mathbf{D} \leftrightarrow\left(\mathbf{E}_{0} \hat{\oplus} \mathbf{E}_{1}\right)=\mathbf{E}\left(N_{0}+N_{1}, p_{d}+p_{e}, q_{d}+q_{e} ; I_{d} I_{e}^{(0)}, J_{d} J_{e}^{(0)}\right) \\
& +\mathbf{E}\left(N_{0}+N_{1}, p_{d}+p_{e}, q_{d}+q_{e} ; I_{d} I_{e}^{(1)}+\left[0^{p_{d}} N_{0}^{p_{e}}\right], J_{d} J_{e}^{(1)}+\left[0^{q_{d}} N_{0}^{q_{e}}\right]\right)
\end{aligned}
$$

Since $n_{a}+n_{b}>p_{e}$, we have

$$
\begin{aligned}
& \mathbf{A} \leftrightarrow\left(\left(\mathbf{B}_{0} \leftrightarrow \mathbf{C}_{0}\right) \hat{\oplus}\left(\mathbf{B}_{1} \leftrightarrow \mathbf{C}_{1}\right)\right) \quad \mathbf{E}\left(N_{0}+N_{1}, m_{a}+m_{b}+m_{c}, n_{a}+n_{b}+n_{c} ; I_{a} I_{b}^{(0)} I_{c}^{(0)}, J_{a} J_{b}^{(0)} J_{c}^{(0)}\right) \\
& \begin{array}{l}
\mathbf{D} \leftrightarrow\left(\mathbf{E}_{0} \hat{\oplus} \mathbf{E}_{1}\right) \\
\mathbf{E}
\end{array} \quad \mathbf{E}\left(N_{0}+N_{1}, p_{d}+p_{e}, q_{d}+q_{e} ; I_{d} I_{e}^{(0)}, J_{d} J_{e}^{(0)}\right) \\
& \mathbf{E}\left(N_{0}+N_{1}, m_{a}+m_{b}+m_{c}, n_{a}+n_{b}+n_{c} ; I_{a} I_{b}^{(1)} I_{c}^{(1)}+\left[0^{m_{a}} N_{0}^{m_{b}+m_{v}}\right], J_{a} J_{b}^{(1)} J_{c}^{(1)}+\left[0^{n_{a}} N_{0}^{n_{b}+n_{v}}\right]\right)
\end{aligned}
$$

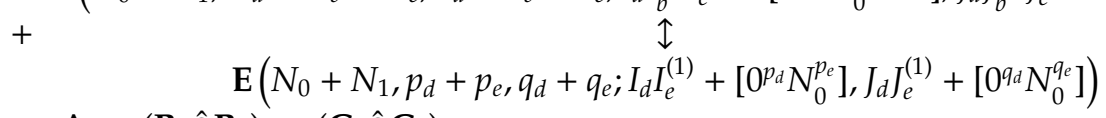

$$
\begin{aligned}
& \mathbf{A} \leftrightarrow\left(\mathbf{B}_{0} \hat{\oplus} \mathbf{B}_{1}\right) \leftrightarrow\left(\mathbf{C}_{0} \hat{\oplus} \mathbf{C}_{1}\right) \\
& =\quad \mathbf{D} \leftrightarrow\left(\mathbf{E}_{0} \hat{\oplus} \mathbf{E}_{1}\right)
\end{aligned}
$$


More generally,

Theorem 2. Let $\mathbf{A}_{\alpha}(\epsilon)=\left(\begin{array}{c}\mathfrak{a}(\alpha, \epsilon) \\ J\end{array}\right)_{I, J} \in \mathbb{K}\left(N_{\epsilon} ; m_{\alpha}, n_{\alpha}\right), \mathbf{B}_{\beta}(\epsilon)=\left(\begin{array}{c}\mathrm{b}(\beta, \epsilon) \\ J\end{array}\right)_{I, J} \in \mathbb{K}\left(N_{\epsilon} ; p_{\beta}, q_{\beta}\right)$, for $\alpha \in\{1, \ldots, k\}$, $\beta \in\{1, \ldots, \ell\}$ and $\epsilon=0,1$ satisfying

1. $p_{1}+\cdots+p_{\beta}=n_{1}+\cdots+n_{\alpha}$ implies $\beta=\ell$ and $\alpha=k$.

2. $n_{\alpha}, p_{\beta}>0$

Then we have

$$
\begin{gathered}
\left(\mathbf{A}_{1}(0) \hat{\oplus} \mathbf{A}_{1}(1)\right) \leftrightarrow \underset{\uparrow}{\cdots}\left(\mathbf{A}_{k}(0) \hat{\oplus} \mathbf{A}_{k}(1)\right) \\
\left(\mathbf{B}_{1}(0) \hat{\oplus} \mathbf{B}_{1}(1)\right) \leftrightarrow \cdots \leftrightarrow\left(\mathbf{B}_{\ell}(0) \hat{\oplus} \mathbf{B}_{\ell}(1)\right)
\end{gathered} \quad=\begin{gathered}
\left(\mathbf{A}_{1}(0) \leftrightarrow \cdots \leftrightarrow \mathbf{A}_{k}(0)\right) \\
\left(\mathbf{B}_{1}(0) \leftrightarrow \cdots \leftrightarrow \mathbf{B}_{\ell}(0)\right)
\end{gathered} \hat{\oplus} \begin{gathered}
\left(\mathbf{A}_{1}(1) \leftrightarrow \cdots \leftrightarrow \mathbf{A}_{k}(1)\right) \\
\left(\mathbf{B}_{1}(1) \leftrightarrow \cdots \leftrightarrow \mathbf{B}_{\ell}(1)\right)
\end{gathered}
$$

Proof. We prove the result by induction on $k+\ell$. The initial case is given by Proposition 6 The condition 1 implies either $p_{1}+\cdots+p_{\ell-1}<n_{1}+\cdots+n_{k-1}$ or $p_{1}+\cdots+p_{\ell-1}>n_{1}+\cdots+n_{k-1}$. The two cases been symmetrical let us consider only the case when $p_{1}+\cdots+p_{\ell-1}<n_{1}+\cdots+n_{k-1}$. Set $\mathbf{A}^{\prime}(\epsilon)=\mathbf{A}_{k-1}(\epsilon) \leftrightarrow \mathbf{A}_{k}(\epsilon)$ for $\epsilon=\{0,1\}$. By induction, we have

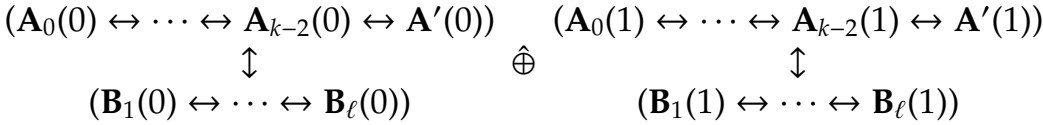

$$
\begin{aligned}
& \left(\mathbf{A}_{0}(0) \hat{\oplus} \mathbf{A}_{0}(1)\right) \leftrightarrow \cdots \leftrightarrow\left(\mathbf{A}_{k-2}(0) \hat{\oplus} \mathbf{A}_{k-2}(1)\right) \leftrightarrow\left(\mathbf{A}^{\prime}(0) \hat{\oplus} \mathbf{A}^{\prime}(1)\right) \\
& =\quad\left(\mathbf{B}_{1}(0) \hat{\oplus} \mathbf{B}_{1}(1)\right) \leftrightarrow \cdots \leftrightarrow\left(\mathbf{B}_{\ell}(0) \hat{\oplus} \mathbf{B}_{\ell}(1)\right)
\end{aligned}
$$

with the notation $\mathbf{A}_{0}(\epsilon)=\mathbf{I}_{0}=1$.

Setting $\mathbf{A}^{\prime \prime}=\left(\mathbf{A}_{0}(0) \hat{\oplus} \mathbf{A}_{0}(1)\right) \leftrightarrow \cdots \leftrightarrow\left(\mathbf{A}_{k-2}(0) \hat{\oplus} \mathbf{A}_{k-2}(1)\right), \mathbf{B}_{\epsilon}^{\prime \prime}=\mathbf{A}_{k-1}(\epsilon), \mathbf{C}_{\epsilon}^{\prime \prime}=\mathbf{A}_{k}(\epsilon), \mathbf{D}^{\prime \prime}=$ $\left(\mathbf{B}_{1}(0) \hat{\oplus} \mathbf{B}_{1}(1)\right) \leftrightarrow \cdots \leftrightarrow\left(\mathbf{B}_{\ell-1}(0) \hat{\oplus} \mathbf{B}_{\ell-1}(1)\right), \mathbf{E}_{\epsilon}^{\prime \prime}=\mathbf{B}_{\ell}(\epsilon), m_{a}=m_{1}+\cdots+m_{k-2}, n_{a}=n_{1}+\cdots+n_{k-2}$, $m_{b}=m_{k-1}, n_{b}=n_{k-1}, m_{c}=m_{k}, n_{c}=n_{k}, p_{d}=p_{1}+\cdots+p_{\ell-1}, q_{d}=q_{1}+\cdots+q_{\ell-1}, p_{e}=p_{\ell}$ and $q_{e}=q_{\ell}$, we obtain

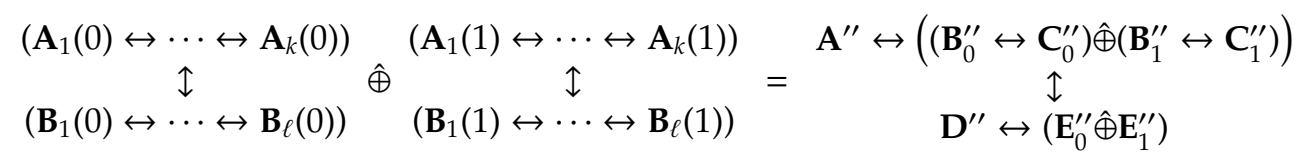

with $m_{a}, n_{a}, m_{b}, m_{c}, p_{d}, q_{d}, q_{e} \geq 0, n_{b}, n_{c}, p_{e}>0, n_{a}+n_{b}>p_{d}$ and $n_{a}+n_{b}+n_{c}=p_{d}+p_{e}$. So by Equation (27), we obtain

$$
\begin{aligned}
& \begin{array}{c}
\left(\mathbf{A}_{1}(0) \leftrightarrow \cdots \leftrightarrow \mathbf{A}_{k}(0)\right) \\
\begin{array}{c}
\uparrow \\
\left(\mathbf{B}_{1}(0) \leftrightarrow \cdots \leftrightarrow \mathbf{B}_{\ell}(0)\right)
\end{array}
\end{array} \quad \begin{array}{c}
\left(\mathbf{A}_{1}(1) \leftrightarrow \cdots \leftrightarrow \mathbf{A}_{k}(1)\right) \\
\hat{\oplus}
\end{array} \quad \begin{array}{c}
\mathbf{A}^{\prime \prime} \leftrightarrow\left(\mathbf{B}_{0}^{\prime \prime} \hat{\oplus} \mathbf{B}_{1}^{\prime \prime}\right) \leftrightarrow\left(\mathbf{C}_{0}^{\prime \prime} \hat{\oplus} \mathbf{C}_{1}^{\prime \prime}\right) \\
\left(\mathbf{B}_{1}(1) \leftrightarrow \cdots \leftrightarrow \mathbf{B}_{\ell}(1)\right)
\end{array} \quad=\begin{array}{c}
\hat{I} \\
\mathbf{D}^{\prime \prime} \leftrightarrow\left(\mathbf{E}_{0}^{\prime \prime} \hat{\oplus} \mathbf{E}_{1}^{\prime \prime}\right)
\end{array} \\
& \begin{aligned}
\left(\mathbf{A}_{0}(0) \hat{\oplus} \mathbf{A}_{0}(1)\right) \leftrightarrow & \cdots \leftrightarrow\left(\mathbf{A}_{k}(0) \hat{\oplus} \mathbf{A}_{k}(1)\right) \\
& \left(\mathbf{B}_{1}(0) \hat{\oplus} \mathbf{B}_{1}(1)\right) \leftrightarrow \\
\stackrel{\cdots}{\uparrow} & \cdots\left(\mathbf{B}_{\ell}(0) \hat{\oplus} \mathbf{B}_{\ell}(1)\right)
\end{aligned} \\
& \begin{aligned}
\left(\mathbf{A}_{1}(0) \hat{\oplus} \mathbf{A}_{1}(1)\right) & \leftrightarrow \cdots \leftrightarrow\left(\mathbf{A}_{k}(0) \hat{\oplus} \mathbf{A}_{k}(1)\right) \\
& \cdots \\
\left(\mathbf{B}_{1}(0) \hat{\oplus} \mathbf{B}_{1}(1)\right) & \leftrightarrow \cdots \leftrightarrow\left(\mathbf{B}_{\ell}(0) \hat{\oplus} \mathbf{B}_{\ell}(1)\right)
\end{aligned}
\end{aligned}
$$

This ends the proof. 


\section{Representations of circuit PROs}

\subsection{Representations of Free PROs}

Let $\mathbb{K}$ be a semiring and $\mathcal{P}$ be a PRO. A multilinear representation of $\mathcal{P}$ is a morphism of $\mathrm{PRO}$ from $\mathcal{P}$ to $\mathbb{K}(N)$. Straightforwardly from the definition of freeness, one has

Claim 2. Any free PRO has multilinear representations.

Remark that the free PRO $\mathcal{F}(\mathcal{X})$ is naturally extended as a $\mathbb{K}$-ModPro $\mathbb{K}\langle\mathcal{X}\rangle:=\bigcup_{p, q \geq 1} \mathbb{K}\left[\mathcal{F}_{p, q}(\mathcal{X})\right]$ where $\mathbb{K}\left[\mathcal{F}_{p, q}(\mathcal{X})\right]$ denotes the free $\mathbb{K}$-module generated by $\mathcal{F}_{p, q}(\mathcal{X})$. A representation $\mu: \mathcal{F}(\mathcal{X}) \rightarrow \mathbb{K}(N)$ induces a morphism of ModPro $\mathbb{K}\langle\mathcal{X}\rangle \rightarrow \mathbb{K}(N)$.

The Hadamard product of two representations $\mu: \mathcal{F}(\mathcal{X}) \rightarrow \mathbb{K}(M)$ and $\mu^{\prime}: \mathcal{F}(\mathcal{X}) \rightarrow \mathbb{K}(N)$ is the unique representation $\mu \widehat{\odot} \mu^{\prime}: \mathcal{F}(X) \rightarrow \mathbb{K}(M N)$ satisfying $\left(\mu \widehat{\odot} \mu^{\prime}\right)(\mathfrak{x})=\mu(\mathfrak{x}) \odot \mu^{\prime}(\mathfrak{x})$ for each $\mathfrak{x} \in \mathcal{X}$.

As a direct consequence of Theorem 1 , we obtain the following result.

Corollary 1. Let $\mu: \mathcal{F}(X) \rightarrow \mathbb{K}(M)$ and $\mu^{\prime}: \mathcal{F}(X) \rightarrow \mathbb{K}(N)$ be two representations of the free PRO. Then for any $\mathfrak{x} \in \mathcal{F}(C),\left(\mu \hat{\odot} \mu^{\prime}\right)(\mathfrak{x})=\mu(\mathfrak{x}) \odot \mu^{\prime}(\mathfrak{x})$.

Proof. The result is proved by induction using equation (20) and (22).

In this section, we focus on representation of Circuit PROs. Consider a set of chips $C=\bigcup_{n, m \geq 1} C_{n, m}$. Any graded map $\mu: C \rightarrow \mathbb{K}(N)$ defines a unique morphism of PROs, $\mu: \operatorname{Circ}(C) \rightarrow \mathbb{K}(N)$.

\subsection{Quasi direct sum of representations of circuit PROs}

The quasi direct sum of two representations $\mu: \operatorname{Circ}(C) \rightarrow \mathbb{K}(M)$ and $\mu^{\prime}: \operatorname{Circ}(C) \rightarrow \mathbb{K}(N)$ is the unique representation $\mu \hat{\oplus} \mu^{\prime}: \operatorname{Circ}(C) \rightarrow \mathbb{K}(M+N)$ satisfying $\left(\mu \hat{\oplus} \mu^{\prime}\right)(\mathfrak{c})=\mu(\mathfrak{c}) \hat{\oplus} \mu^{\prime}(\mathfrak{c})$ for each $\mathfrak{c} \in C$.

Note that in general

$$
\left(\mu \hat{\oplus} \mu^{\prime}\right)\left(\mathfrak{c} \leftrightarrow \mathfrak{c}^{\prime}\right) \neq \mu\left(\mathfrak{c} \leftrightarrow \mathfrak{c}^{\prime}\right) \hat{\oplus} \mu^{\prime}\left(\mathfrak{c} \leftrightarrow \mathfrak{c}^{\prime}\right) .
$$

Example 4. Let $\mu^{0}$ and $\mu^{1}$ be two 2-dimensional representations. One has

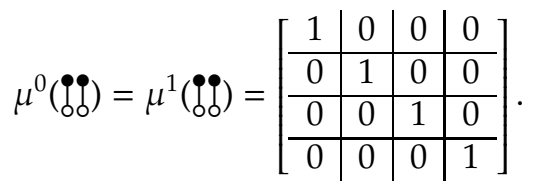

Hence,

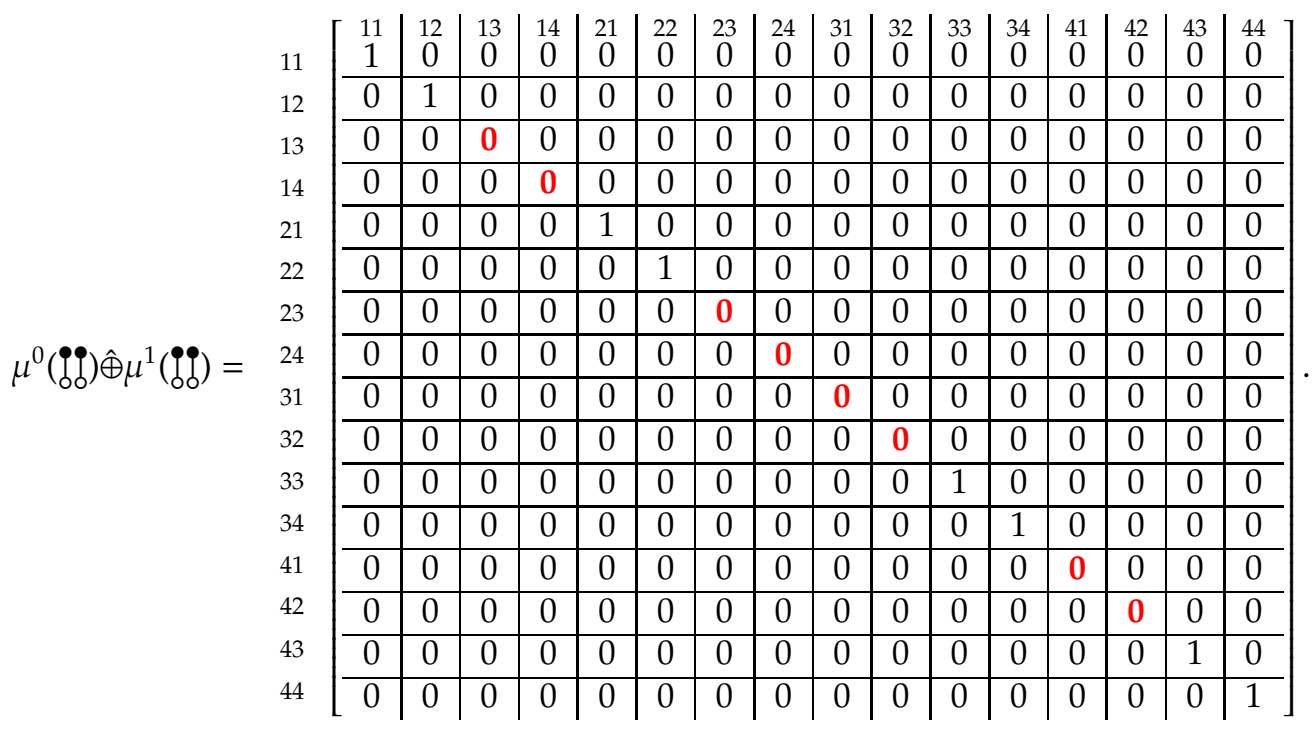


Whilst

$$
\mu^{0} \hat{\oplus} \mu^{1}(\stackrel{\mathfrak{o}}{\mathfrak{l}})=\mu^{0} \hat{\oplus} \mu^{1}(\stackrel{\mathfrak{l}}{)}) \leftrightarrow \mu^{0} \hat{\oplus} \mu^{1}(\mathfrak{l})=\left(\begin{array}{l}
I \\
\delta \\
J
\end{array}\right)_{I \in[4]^{2}, J \in[4]^{2}} .
$$

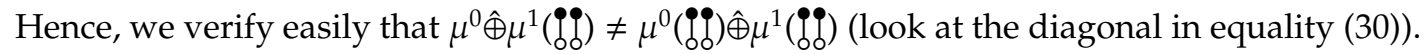

Nevertheless, Proposition \implies that if $\left(\mu \hat{\oplus} \mu^{\prime}\right)(\mathfrak{c})=\mu(\mathfrak{c}) \hat{\oplus} \mu^{\prime}(\mathfrak{c})$ and $\left(\mu \hat{\oplus} \mu^{\prime}\right)\left(\mathfrak{c}^{\prime}\right)=\mu\left(\mathfrak{c}^{\prime}\right) \hat{\oplus} \mu^{\prime}\left(\mathfrak{c}^{\prime}\right)$ then

$$
\left(\mu \hat{\oplus} \mu^{\prime}\right)\left(\begin{array}{c}
\mathfrak{c} \\
\mathfrak{I} \\
\mathfrak{c}^{\prime}
\end{array}\right)=\mu\left(\begin{array}{c}
\mathfrak{c} \\
\mathfrak{1} \\
\mathfrak{c}^{\prime}
\end{array}\right) \hat{\oplus} \mu^{\prime}\left(\begin{array}{c}
\mathfrak{c} \\
\mathfrak{I} \\
\mathfrak{c}^{\prime}
\end{array}\right) \text {. }
$$

More generally on has

Theorem 3. Let $\mu^{0}: \operatorname{Circ}(C) \rightarrow \mathbb{K}\left(N_{0}\right)$ and $\mu^{1}: \operatorname{Circ}(C) \rightarrow \mathbb{K}\left(N_{1}\right)$ be two representations. For any connected circuit $\mathrm{c} \in \operatorname{Circ}(C)_{m, n}$, one has

$$
\left(\mu^{0} \hat{\oplus} \mu^{1}\right)(\mathfrak{c})=\mu^{0}(\mathfrak{c}) \hat{\oplus} \mu^{1}(\mathfrak{c})
$$

Proof. We prove the result by induction following the point 3 of Proposition 2

If $\mathfrak{c}$ is a chip then the property is straightforward from the definition.

If $\mathfrak{c}=\boldsymbol{i}$ then $\mu^{0}(\mathfrak{l})=\mathbf{I}\left(N_{0}\right), \mu^{1}(\stackrel{\mathfrak{l}}{)})=\mathbf{I}\left(N_{1}\right)$ and

$$
\left(\mu^{0} \hat{\oplus} \mu^{1}\right)(\mathfrak{\wp})=\mathbf{I}\left(N_{0}+N_{1}\right)=\mathbf{I}\left(N_{0}\right) \hat{\oplus} \mathbf{I}\left(N_{1}\right)=\mu^{0}(\mathfrak{l}) \hat{\oplus} \mu^{1}(\mathfrak{l})
$$

from equality (23).

For the other cases, $\mathfrak{c}$ admits an expression as

$$
\mathfrak{c}=\begin{gathered}
\mathfrak{p}_{1} \leftrightarrow \cdots \leftrightarrow \mathfrak{p}_{k} \\
\mathfrak{q} \\
\mathfrak{q}_{1} \leftrightarrow \cdots \leftrightarrow \mathfrak{q}_{\ell}
\end{gathered},
$$

such that for each $1 \leq i \leq k$ and $1 \leq j \leq \ell, \mathfrak{p}_{i} \in \operatorname{Circuit}(C)_{m_{i}, n_{i}}$ and $\mathfrak{q}_{j} \in \operatorname{Circ}(C)_{p_{j}, q_{j}}$ are connected circuits and $n_{1}+\cdots+n_{i}=p_{1}+\cdots p_{j}$ implies $i=k$ and $j=\ell$. By induction, for each $i$ and $j$, $\left(\mu^{0} \hat{\oplus} \mu^{1}\right)\left(\mathfrak{p}_{i}\right)=\mu^{0}\left(\mathfrak{p}_{i}\right) \hat{\oplus} \mu^{1}\left(\mathfrak{p}_{i}\right)$ and $\left(\mu^{0} \hat{\oplus} \mu^{1}\right)\left(\mathfrak{q}_{j}\right)=\mu^{0}\left(\mathfrak{q}_{j}\right) \hat{\oplus} \mu^{1}\left(\mathfrak{q}_{j}\right)$.

Hence,

$$
\left(\mu^{0} \hat{\oplus} \mu^{1}\right)(c)=\begin{gathered}
\mu^{0}\left(\mathfrak{p}_{1}\right) \hat{\oplus} \mu^{1}\left(\mathfrak{p}_{1}\right) \leftrightarrow \\
\begin{array}{c}
\cdots \\
\mathfrak{1}
\end{array} \\
\mu^{0}\left(\mathfrak{q}_{1}\right) \hat{\oplus} \mu^{1}\left(\mathfrak{q}_{1}\right) \leftrightarrow \cdots \leftrightarrow \mu^{0}\left(\mathfrak{p}_{k}\right) \hat{\oplus} \mu^{1}\left(\mathfrak{p}_{k}\right)
\end{gathered} .
$$

From Theorem 2 we obtain

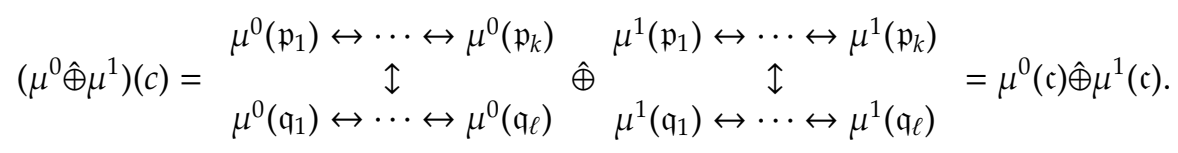

\subsection{From paths to representations}

Let $\mu: \operatorname{Circ}(C) \longrightarrow \mathbb{K}(N)$ be a representation of a circuit PRO. We denote by $\underset{J}{\mu(\mathfrak{p})}$ the entries of the hypermatrix $\mu(\mathfrak{p})$ for any circuit $\mathfrak{p} \in \mathcal{F}(\mathcal{X})$.

We define the map $\tilde{\mu}: \mathcal{P}$ aths $(C, N) \longrightarrow \mathbb{K}$ by 


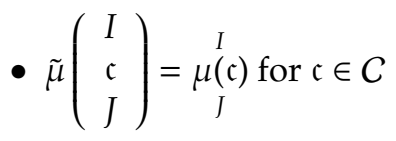

- $\tilde{\mu}\left(\begin{array}{l}\mathfrak{p} \\ \mathfrak{l} \\ \mathfrak{q}\end{array}\right)=\tilde{\mu}(\mathfrak{p}) \tilde{\mu}(\mathfrak{q})$ when $\operatorname{Out}(\mathfrak{q})=\operatorname{In}(\mathfrak{p})$.

- $\tilde{\mu}\left(\mathfrak{p} \leftrightarrow \mathfrak{p}^{\prime}\right)=\tilde{\mu}(\mathfrak{p}) \tilde{\mu}\left(\mathfrak{p}^{\prime}\right)$.

Proposition 7. We have

$$
\underset{J}{\stackrel{I}{\mu}(\mathfrak{p})}=\sum \tilde{\mu}(\mathfrak{q})
$$

where the sum is over the paths $\mathfrak{q} \in \mathcal{P}$ aths $(C, N)$ such that $u(\mathfrak{q})=\mathfrak{p}$, Out $(\mathfrak{q})=I$, and $\operatorname{In}(\mathfrak{q})=J$.

Proof. We prove the result by induction. We have to consider three cases

- If $\mathfrak{p} \in C$ then the proposition is straightforward from the definition.

- If $\mathfrak{p}=\mathfrak{p}_{1} \leftrightarrow \mathfrak{p}_{2}$, with $\mathfrak{p}_{1} \in \operatorname{Circuit}(C)_{m_{1}, n_{1}}, \mathfrak{p}_{2} \in \operatorname{Circuit}(C)_{m_{2}, n_{2}}$ and $\mathfrak{p}_{1}, \mathfrak{p}_{2} \neq \mathfrak{p}$, then

$$
\mu\left(\mathfrak{p}_{1} \underset{J_{1} I_{2}}{\stackrel{I_{1}}{\leftrightarrow}} \mathfrak{p}_{2}\right)=\underset{J_{1}}{\stackrel{I_{1}}{\mu} \underset{\mathfrak{p}_{1}}{\stackrel{I_{2}}{I_{2}}} \mu\left(\mathfrak{p}_{2}\right)}
$$

for any $I_{1} \in[N]^{m_{1}}, I_{2} \in[N]^{m_{2}}, I_{1} \in[N]^{n_{1}}$, and $J_{2} \in[N]^{n_{2}}$. By induction, one obtains

$$
\underset{J_{1}}{\stackrel{I_{1}}{\mu}} \underset{\mathfrak{p}_{1}}{I_{J_{2}}} \leftrightarrow \underset{\mathrm{I}_{2}}{\mu}\left(\mathfrak{p}_{2}\right)=\sum \tilde{\mu}\left(\mathfrak{q}_{1}\right) \tilde{\mu}\left(\mathfrak{q}_{2}\right)=\sum \tilde{\mu}\left(\mathfrak{q}_{1} \leftrightarrow \mathfrak{q}_{2}\right)
$$

where the sum is over the pairs of paths $\mathfrak{q}_{1}, \mathfrak{q}_{2} \in \mathcal{P}$ aths $(C, N)$ satisfying $u\left(\mathfrak{q}_{1}\right)=\mathfrak{p}_{1}$, Out $\left(\mathfrak{q}_{1}\right)=I_{1}$, $\operatorname{In}\left(\mathfrak{q}_{1}\right)=J_{1}, u\left(\mathfrak{q}_{2}\right)=\mathfrak{p}_{2}, \operatorname{Out}\left(\mathfrak{q}_{2}\right)=I_{2}$, and $\operatorname{In}\left(\mathfrak{q}_{2}\right)=J_{2}$. We deduce our result from Lemma 2

- If $\mathfrak{p}=\underset{\substack{\mathfrak{p}^{\prime} \\ \mathfrak{p}}}{\mathfrak{p}^{\prime}}$ with $\mathfrak{p}^{\prime} \in \operatorname{Circ}(C)_{m, n}, \mathfrak{p}^{\prime \prime} \in \operatorname{Circ}(C)_{n, p}$, and $\mathfrak{p}^{\prime}, \mathfrak{p}^{\prime \prime} \neq \mathfrak{p}$, then one has

$$
\left.\underset{J}{I}(\mathfrak{p})=\sum_{K} \underset{K}{\mu} \underset{I}{I} \mathfrak{p}^{\prime}\right) \underset{I}{\left.\stackrel{K}{\left(\mathfrak{p}^{\prime \prime}\right.}\right)}
$$

By induction, one has

$$
\underset{J}{\stackrel{I}{(p)}}=\sum_{K} \sum_{\mathfrak{q}^{\prime}, q^{\prime \prime}} \tilde{\mu}\left(\mathfrak{q}^{\prime}\right) \tilde{\mu}\left(\mathfrak{q}^{\prime \prime}\right)
$$

where the second sum is over the pairs of paths $\mathfrak{q}^{\prime}, \mathfrak{q}^{\prime \prime} \in \mathcal{P}$ aths $(C, N)$ satisfying $u\left(\mathfrak{q}^{\prime}\right)=\mathfrak{p}^{\prime}$, Out $\left(\mathfrak{q}^{\prime}\right)=$ $I, \operatorname{In}\left(\mathfrak{q}^{\prime}\right)=K, u\left(\mathfrak{q}^{\prime \prime}\right)=\mathfrak{p}^{\prime \prime}, \operatorname{Out}\left(\mathfrak{q}^{\prime \prime}\right)=K$, and $\operatorname{In}\left(\mathfrak{q}^{\prime \prime}\right)=J$. Hence,

$$
\underset{J}{I}(\mathfrak{p})=\sum_{\mathfrak{q}^{\prime}, \mathfrak{q}^{\prime \prime}} \tilde{\mu}\left(\begin{array}{c}
\mathfrak{q}^{\prime} \\
\uparrow \\
\mathfrak{q}^{\prime \prime}
\end{array}\right)
$$

where the sum is over the pairs of paths $\mathfrak{q}^{\prime}, \mathfrak{q}^{\prime \prime} \in \mathcal{P}$ aths $(C, N)$ satisfying $u\left(\mathfrak{q}^{\prime}\right)=\mathfrak{p}^{\prime}$, $\operatorname{Out}\left(\mathfrak{q}^{\prime}\right)=I$, $u\left(\mathfrak{q}^{\prime \prime}\right)=\mathfrak{p}^{\prime \prime}, \operatorname{Out}\left(\mathfrak{q}^{\prime \prime}\right)=\operatorname{In}\left(\mathfrak{q}^{\prime}\right)$, and $\operatorname{In}\left(\mathfrak{q}^{\prime \prime}\right)=J$. Again, Lemma 2 allows us to conclude. 
Example 5. Let $C=C_{1,2} \cup C_{2,2}$ with $C_{2,2}=\{\square\}$ and $C_{1,2}=\{\square\}$. Let $\mu: \operatorname{Circuit}(C) \longrightarrow \mathbb{K}(3)$ be the representation satisfying

- $\mu($ a $)=\mathbf{A}$ where $\underset{2,3}{\stackrel{2,3}{\mathbf{A}}}=x, \underset{2,3}{\mathrm{~A}, 3}=x^{\prime}$ and $\underset{i, j}{\mathrm{k}, l}=0$ otherwise.

- $\mu(\mathbb{b})=B$ where $\underset{2}{\mathbf{B}}=y, \underset{3}{\mathbf{B}}=y^{\prime}$, and $\underset{i}{\mathbf{B}}=0$ in the other cases.

Proposition 7 means that the representation $\mu$ can be graphically represented by an hypergraph as in figure 6, where only the non zero transition are drawn, and that the image of circuit is obtained by summing over all the generalized paths.

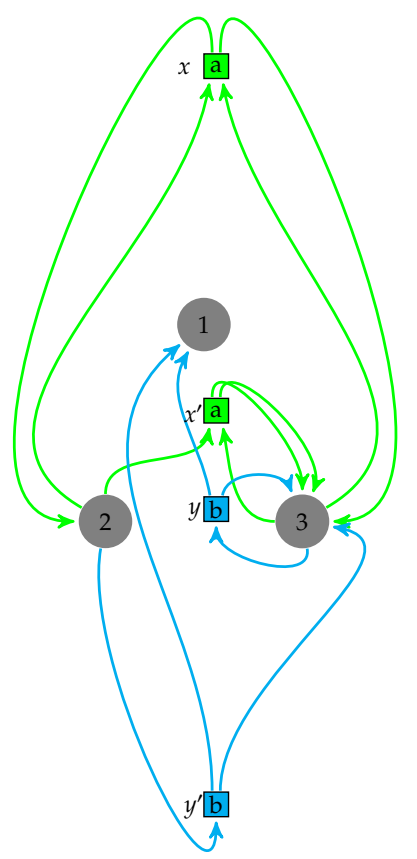

Figure 6: An example of hypergraph.

For instance, we consider the following entry:

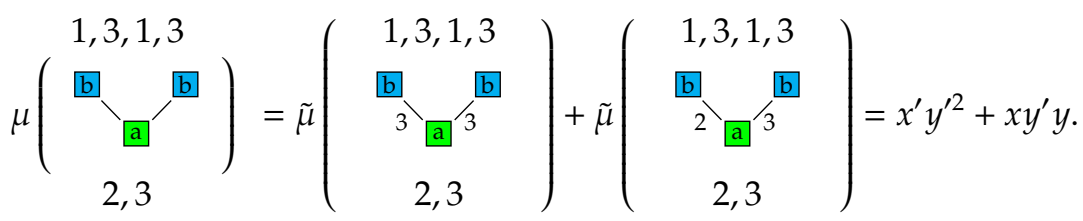




\section{Toward a universal definition of automata}

Finite state automata belong to the large class of abstract machines studied in the context of information theory and formal languages. In particular, they play an important role in the Chomsky hierarchy [8, 9] since they characterize regular languages [18]. More precisely, automata provide an acceptance mechanism for words and the set of the words accepted by a given automaton is its recognized language. According to Kleene's theorem [18] the set of languages which are recognized by automata is the smallest set containing the subsets of symbols and closed by union, concatenation and star. The first occurrence of this concept in literature dates back to the paper of McCulloch and Pitt [25] but the notion of non deterministic finite automaton (NFA) was formally introduced by Rabin and Scott [26]. An automaton is a graph based machine with states and transitions labeled with letters. More precisely, an automaton is a quintuple $(\Sigma, Q, I, F, \delta)$ where $\Sigma$ is the alphabet labeling transitions, $Q$ is the set of the states, some of states are called initial $(I \subset Q)$, some others are called final $(F \subset Q)$, and $\delta$ is the transition function which sends each pair of $Q \times \Sigma$ to a subset of $Q$. A word is recognized by an automaton if starting from an initial state, one can reach a final state following a path labeled with the sequence of the letters of the word. In order to introduce weight on transitions, it is easier to consider automata as linear representations [27]. More precisely, an automaton with weight taken in a semiring $\mathbb{K}$ is a triplet $\mathrm{A}=(\lambda, \rho, \gamma)$ where $\lambda \in \mathbb{K}^{1 \times N}, \gamma \in \mathbb{K}^{N \times 1}$ and $\rho$ is a morphism of monoid from the free monoid $\Sigma^{*}$ to $\mathbb{K}^{N \times N}$, each $\rho(a)$, for $a \in \Sigma$, is the adjacency matrix associated to the weighted subgraph whose edges are labeled with $a$ ( $N$ is the number of states and $\rho$ encodes the transitions). The automaton A associates to each word $w \in \Sigma$ the scalar $\lambda \rho(w) \gamma \in \mathbb{K}$. This number is also the sum of all the value obtained by reading the paths labeled with $w$. Weighted tree automata [15] are another example of finite state machines but instead of produce a scalar from a word, a tree automaton associates a scalar to each tree. There exist two kind of tree automata: bottom-up and top-down depending on the trees being read from the leaves to the root or the root to the leaves. In both cases, the computation has in commons with that of classical automaton the fact that it is based on both (multi)linear representations and an appropriate notion of paths.

However, the history of the theory of finite automata begins long before the work of postwar pioneers. Indeed, one of the important sources of inspiration was the theory of electronic circuits as it was studied from the nineteenth century. Graphically, a finite automaton is very closed to an electronic circuit: the states of the automaton play the same role as the nodes of a circuit, the transitions the same role as electric components and the letter labeling the transition refer to the type of the component connecting the two nodes. To continue the analogy, the letters of an alphabet symbolize components with only one input and one output. The letters in a tree automaton can be considered as one input/ many output or many input/ one output components depending on the automata being bottom-up or top-down.

The goal of this section is to show that multilinear representations of PROs underly in the definition of many kind of automata.

\subsection{Word automata}

Suppose that $\mathcal{K}$ is a commutative semiring. Recall that a word automata is a triplet A $:=(\lambda, \rho, \gamma)$ where $\lambda \in \mathbb{K}^{1 \times N}, \gamma \in \mathbb{K}^{N \times 1}$, and $\rho$ is a morphism from a free monoid $A^{*}$ to $\mathbb{K}^{N \times N}$. The behavior of A is the series $B_{\mathrm{A}}=\sum_{w \in A^{*}} \lambda \rho(w) F \gamma w$. Since $\mathbb{K}$ is commutative, this kind of automaton is easily mimicked through a multilinear representation of PRO. First we consider the free PROs $\mathcal{F}(\mathcal{X})$ generated by $\mathcal{X}=\mathcal{X}_{1,0} \cup \mathcal{X}_{1,1} \cup \mathcal{X}_{0,1}$ with $\mathcal{X}_{1,0}=\{\square\}, \mathcal{X}_{1,1}=\{\square: a \in A\}$ and $\mathcal{X}_{0,1}=\{\square\}$. We consider also representation of PROs $\mu: \mathcal{F}(\mathcal{X}) \rightarrow \mathcal{K}(N)$ defined by $\mu(\square)=\lambda, \mu(\mathbb{\square})=\gamma$ and $\mu(\square)=\rho(a)$. We assimilate a word to an element of $\mathcal{F}(\mathcal{X})$ through the correspondence 


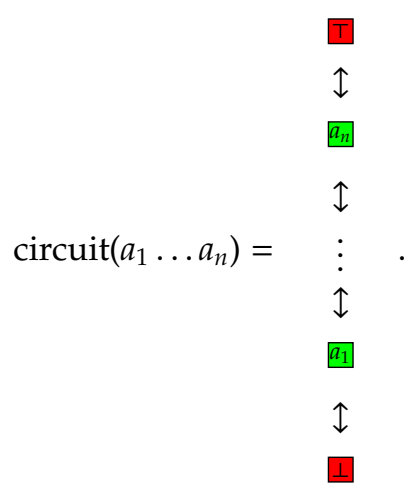

With such a notation, the behavior becomes

$$
B_{\mathrm{A}}=\sum_{w \in A^{*}} \mu(\operatorname{circuit}(w)) w
$$

A formal series is recognizable if it is the behavior of an automaton. It is well known that if two series $S=\sum_{w} \alpha_{w} w$ and $S^{\prime}=\sum_{w} \alpha_{w}^{\prime} w$ are recognizable then their sum $S+S^{\prime}=\sum_{w}\left(\alpha_{w}+\alpha_{w}^{\prime}\right) w$ and Hadamard product $S \odot S^{\prime}=\sum_{w}\left(\alpha_{w} \alpha_{w}^{\prime}\right) w$ are also recognizable. This can be seen as a consequence of the structure of PRO. Indeed, since both series are recognizable, one associates a representation $\mu$ with $S$ and a representation $\mu^{\prime}$ to $S^{\prime}$. From Corollary1, one has

$$
S \odot S^{\prime}=\sum_{w}\left(\mu(\operatorname{circuit}(w)) \mu^{\prime}(\operatorname{circuit}(w)) w=\sum_{w}\left(\mu \odot \hat{\rho} \mu^{\prime}(\operatorname{circuit}(w)) w .\right.\right.
$$

Applying Theorems 2 and 3 , one obtains

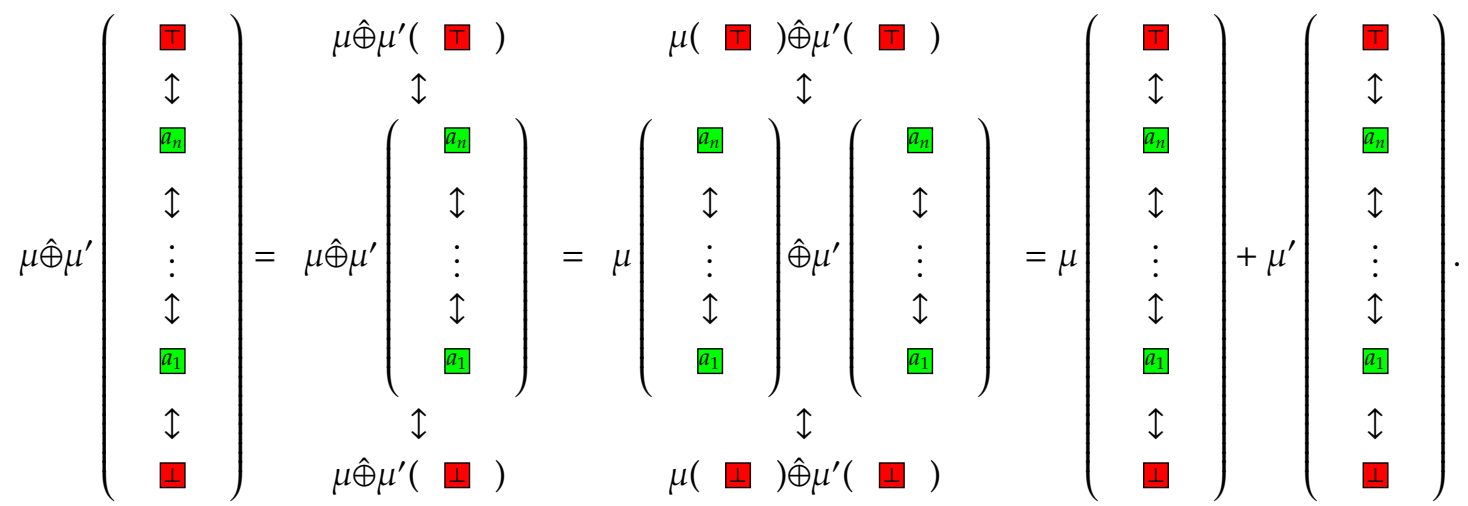

Hence,

$$
S+S^{\prime}=\sum_{w} \mu \hat{\oplus} \mu^{\prime}(\operatorname{circuit}(w)) w
$$

as expected.

\subsection{Tree automata}

For the sake of simplicity we suppose that $\mathcal{K}=\mathbb{B}$ the boolean semiring but all the theory is transposable for any other commutative semiring. Recall that there exist two kind of tree automata: Bottom-Up and Top-Down. The two constructions being symmetrical, we consider here only the Bottom-Up automata. A Bottom-Up automaton (see eg [10]) is a tuple $A=(Q, \Sigma, \delta, F)$ where 
- $Q$ is a finite set of states (without loss of generality we assume $Q=\{1, \ldots, N\}$ for some integer $N$,

- $\Sigma=\cup_{k} \Sigma^{(k)}$ is the ranked input alphabet

- $\delta: \Sigma(Q) \rightarrow 2^{Q}$ is the transition function, where $\Sigma(Q)=\left\{\begin{array}{c}q_{1} q_{2} \ldots q_{k} \\ \prod_{a} /\end{array}: k \in \mathbb{N}, a \in \Sigma^{(k)}, q_{1}, \ldots, q_{k} \in Q\right\}$.

- $F \subseteq Q$ is the set of final states.

For a tree $t=\bigvee_{a}^{t_{1} t_{2} \cdots t_{k}}$, we define

$$
\delta^{*}(t)=\bigcup_{q_{1} \in \delta^{*}\left(t_{1}\right), \ldots, q_{k} \in \delta^{*}\left(t_{k}\right)} \delta\left(\begin{array}{c}
q_{1} q_{2} \ldots q_{k} \\
\bigcup_{a} /
\end{array}\right) .
$$

The language accepted by $A$ is defined by $L(A):=\left\{t: \delta^{*}(t) \cap F \neq \emptyset\right\}$. Notice that a set is nothing but a formal series with multiplicities in $\mathbb{B}$. So,

$$
L(\mathrm{~A})=\sum_{t: \delta^{*}(t) \cap F \neq \emptyset} t .
$$

We construct a free PRO together with a multilinear representation mimicking the behavior of the automaton. We consider the bigraded set $\mathcal{X}=\mathcal{X}_{1,0} \cup \mathcal{X}_{0,1} \cup \cup_{m, 1}$ with $\mathcal{X}_{k, 1}=\left\{a: a \in \Sigma^{(a)}\right\}$, $\mathcal{X}_{1,0}=\{\square\}$, and $\mathcal{X}_{0,1}=\{\square\}$. To each tree we associate a an element of the free Pro $\mathcal{F}(\mathcal{X})$ by setting

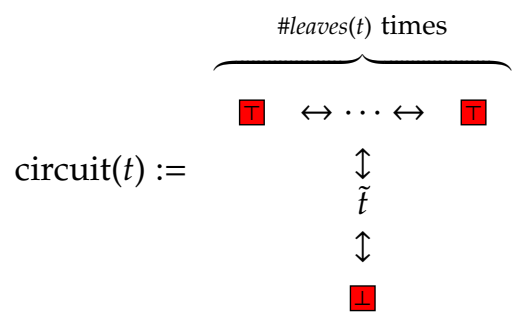

where leaves $(t)$ denotes the set of the leaves of $t$ and $\tilde{t}$ is the element of $\mathcal{F}(\mathcal{X})$ obtained by substituting each occurrence of letters in $\Sigma^{(k)}$ by its corresponding symbol in $\mathcal{X}_{k, 1}$. For instance,

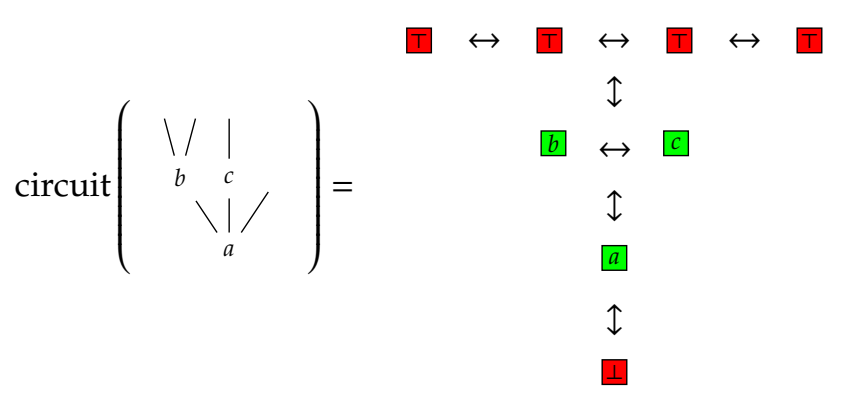

Now we define the representation $\mu$ satisfying

- $\mu(\perp):=1$, 
- $\mu(\mathbb{T})=1$ if $q \in F$ and 0 otherwise,

- for each $a \in \Sigma^{(k)}$, we define $\mu(\square)$ such that $\left.\begin{array}{c}q_{1}, \ldots, q_{k} \\ \mu\left(\square^{a}\right) \\ q\end{array}\right)=1$ if $q \in \delta\left(\begin{array}{c}q_{1} q_{2} \ldots q_{k} \\ \bigvee_{a} /\end{array}\right)$ and 0 otherwise.

With this notation one has

$$
L(\mathrm{~A})=\sum_{t} \mu(\operatorname{circuit}(t)) t
$$

In terms of series, the union of two languages is translated as the sum of the series while the intersection is the Hadamard product. As in the case of the words, if $L$ and $L^{\prime}$ are two languages recognized by automata then $L \cup L^{\prime}$ and $L \cap L^{\prime}$ are also recognized. This also can be seen as consequences of Theorems 3] and 1

\subsection{Branching automata}

Branching automata are a generalisation of usual (Kleene) automata introduced by Lodaya and Weil [21, 22] in the aim to take into account both sequentiality and parallelism. Notice that, this kind of automata have been recently connected to a logic named Presburger-MSO [1], as expressive as branching automata. Recall first that a branching automata is a tuple $\mathrm{A}=(Q, \Sigma, E, I, F)$ where $\Sigma$ is an alphabet, $Q$ is the set of the states, $I, F \subset Q$ are respectively the set of initial and final states, and the transition $E$ splits into $E=\left(E_{\text {seq }}, E_{\text {fork }}, E_{\text {joint }}\right)$ with

- $E_{\text {seq }}$ are usual transitions.

- $E_{\text {fork }} \subset Q \times \mathcal{M}^{>1}(Q)$ and $E_{\text {joint }} \subset \mathcal{M}^{>1}(Q) \times Q$ where $\mathcal{M}^{>1}(Q)$ denotes the set of multi-sets of $Q$ with at least two elements.

We construct a representation of a free PRO in $\mathbb{B}(N)$. We consider the multiset $\mathcal{X}=\mathcal{X}_{1,0} \cup \mathcal{X}_{0,1} \cup \mathcal{X}_{1,1} \cup$ $\bigcup_{n \geq 2} \mathcal{X}_{1, n} \cup \bigcup_{m \geq 2} \mathcal{X}_{m, 1}$ with $\mathcal{X}_{1,0}=\{\square\}, \mathcal{X}_{0,1}=\{\square\}, \mathcal{X}_{1,1}:=\{\square: a \in \Sigma\}, \mathcal{X}_{n, 1}:=\{\bar{n}\}$, and $\mathcal{X}_{1, m}:=\{\underline{\underline{m}}\}$. For $\mathfrak{x} \in \mathcal{X}_{1,0} \cup \mathcal{X}_{0,1} \mathcal{X}_{1,1}, \mu(\mathfrak{x})$ is defined as in the case of word automata. For each $m, n \geq 2$, the hypermatrices $\mu(\sqrt{n})$ and $\mu(\underline{\underline{m}})$ are symmetric in the sense that they satisfy

$$
\begin{aligned}
& i_{1}, \ldots, i_{n} \quad i_{\sigma(1)}, \ldots, i_{\sigma(n)} \quad i \quad i \\
& \mu(\bar{n})=\mu(\bar{n}) \text { and } \mu(\underline{\underline{m}})=\mu(\underline{\underline{m}}) \\
& i \quad i \quad i_{1}, \ldots, i_{m} \quad i_{\tau(1)}, \ldots, i_{\tau(m)} \\
& i_{1}, \ldots, i_{n}
\end{aligned}
$$

for each permutations $\sigma \in \mathfrak{S}_{n}$ and $\tau \in \mathfrak{S}_{m}$. Furthermore, $\mu(\bar{n})=1$ if $\left(i,\left\{i_{1}, \ldots, i_{n}\right\}\right) \in E_{\text {fork }}$ and 0

i

otherwise, and $\mu(\underline{\underline{m}})=1$ if $\left(\left\{i_{1}, \ldots, i_{m}\right\}, i\right) \in E_{\text {joint }}$ and 0 otherwise. For this kind of automata, the $i_{1}, \ldots, i_{m}$

recognized language is assimilated to the formal series

$$
L^{\prime /}(\mathrm{A})=\sum_{\mathfrak{c} \in \mathcal{F}\left(X_{1,1}\right.} \mu\left(\begin{array}{c}
\square \\
\mathfrak{\downarrow} \\
\mathfrak{c} \\
\mathfrak{\downarrow} \\
\square
\end{array}\right) c
$$


Also notice that the notion of path in this model of automata coincides with the notion of path in the hypergraph associated to a representation. Closure under finite union (resp. finite intersection) of languages recognized by branching automata can be seen as a consequence of Theorem 3 (resp. Corollary 1).

Example 6. Consider the branching automaton

$$
\mathrm{A}=([1,6], a, b,(\{(2, a, 4),(3, b, 5)\},\{(1,\{1,1\}),(1,\{2,3\})\},\{(\{6,6\}, 6),(\{4,5\}, 6)\}),\{1\},\{1,6\}) .
$$

The hypergraph of the associated representation is drawn in figure 7 After removing the symbols , $\square,\left[\overline{2}\right.$, and $\left[2\right.$ in the language $L^{/ /}$we obtain exactly all the elements under the forms $a_{1} \leftrightarrow \cdots \leftrightarrow a_{2 n}$ for $n \in \mathbb{N}$ and $a_{i} \in\{a, b\}$ such that $\operatorname{card}\left\{i: a_{i}=a\right\}=\operatorname{card}\left\{i: a_{i}=b\right\}$.

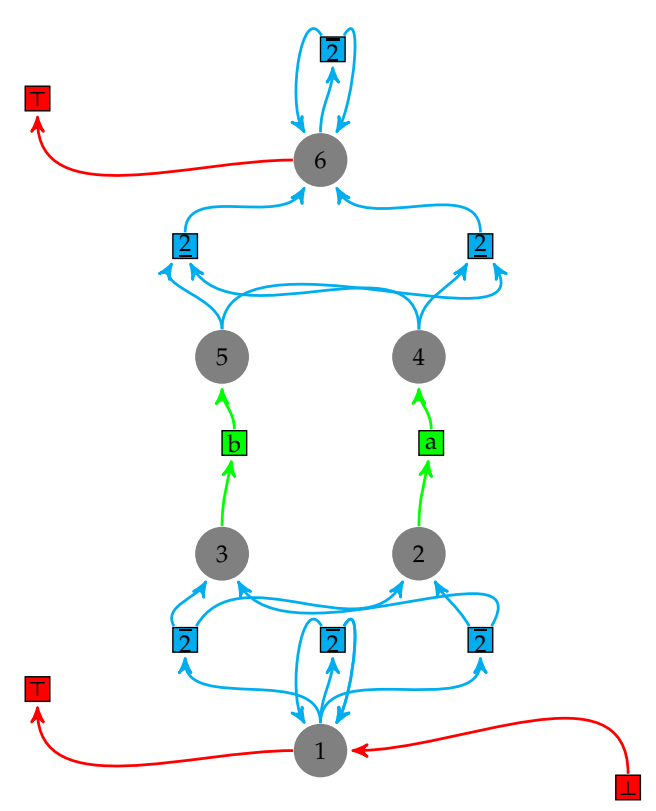

Figure 7: An example of branching automaton seen as the hypergraph of a representation.

\subsection{Heindel Pro automata}

Recently, Heindel [17] proposed a definition for PRO-automata. In his paper, he used a more general definition for PROs (category in Mon whose object monoid is free) which contains PROs and colored PROs as special cases. He used also a definition of free PROs (called placids) which matches to free colored PROs in our notations. To be more precise, he defined the notion of signature associated with a list of colors $C$, as a triplet $(\Sigma, s, t)$ where $\Sigma$ is a finite set and $s: \Sigma \rightarrow C^{*}$ and $t: \Sigma \rightarrow C^{*}$ are two maps which associate to each symbol $\sigma \in \Sigma$ a sequence of inputs $s(\sigma) \in C^{*}$ and a sequence of outputs $t(\sigma) \in C^{*}$. A signature is seen as a graph whose vertices belong to a subset of $C^{*}$ and the arrows are in $\Sigma$. A generalised PRO $\mathcal{D}$ is seen as graph $\left(D_{0}, D_{1}\right)$ where $D_{0}$ is the set of the objects and $D_{1}$ the set of the arrows. The set $D_{0}$ endowed with the tensor product is a monoid and to each arrow $a$ in $D_{1}$ we associate its domain (inputs) $\operatorname{dom}(a) \in D_{0}$ and its codomain (outputs) codom $(a) \in D_{0}$. The definition of a signature embedding generalizes the notion of alphabet in the senses that it is a morphism of graphs 
from a signature $(\Sigma, s, t)$ to a generalized PRO $\left(D_{0}, D_{1}\right)$ together with a morphism of monoid $C^{*} \rightarrow D_{0}$. Let us recall the definition of a placid as a free (generalized) PRO [17]. If $C \neq \emptyset$ be a set of colors and $(\Sigma, s, t)$ be a signature. There exists a placid $\mathcal{P}(\Sigma)$ into which the signature $\Sigma$ embeds via the inclusion $\Sigma \subset \mathcal{P}(\Sigma)$ such that for each generalized PRO $\mathcal{D}$ and embedding $\iota: \Sigma \rightarrow \mathcal{D}$, there exists a unique (generalized) PRO-morphism $F_{\iota}: \mathcal{P}(\Sigma) \rightarrow \mathcal{D}$ that restrict to $\iota$. From this definition, we notice that a placid is nothing but a Path PRO (see section 3.3).

Now we have all the ingredients to recall the definition of PRO automata by [17].

Definition 3. (Heindel [17]) A PRO automaton over the signature $C$-colored signature $(\Sigma, s, t)$ is a tuple $\mathcal{A}=(Q, \Gamma, \delta, I, F)$ where $Q$ is a finite set of states, $\left(\Gamma, s^{\prime}, t^{\prime}\right)$ a signature over the set of colors $Q, I$ and $F$ are two subset of words in $Q^{*}$, and $\delta$ is a morphism of signatures (that is a morphism of graph induced by a morphism of monoid $Q^{*} \rightarrow C^{*}$ ).

For our purpose we assume three restrictions:

- first \#C = 1 this means that the placid $\mathcal{P}(\Sigma)$ is nothing but the free $\operatorname{PRO} \mathcal{F}(\Sigma)$,

- all the elements of $\Sigma$ has at least one input and at least one output,

- the map $s^{\prime} \times \delta_{1} \times t^{\prime}: \Gamma \rightarrow Q^{*} \times \Sigma \times Q^{*}$ is injective, where $\delta_{1}$ denotes the component of the map $\delta$ sending the arrows of the graph $\Gamma$ to the arrows of $\Sigma$, that is the PRO automaton is normal in the sense of [17].

Definition 4. (Heindel [17]) $A$ run of an automaton $\mathcal{A}=(A, \Gamma, \delta, I, F)$ is an arrow in $r: q \rightarrow p$ in $\mathcal{P}(\Gamma)$ and it is accepting if its $q \in I$ and $q^{\prime} \in F$. The language of $\mathcal{A}$ is the set of the image in $\mathcal{F}(\Sigma)$ of the accepting runs. With our notation we have

$$
\mathcal{L}(\mathcal{A})=\{u(\delta(a)) \mid a \in F(\Gamma), \operatorname{In}(a) \in I, \operatorname{Out}(a) \in J\}
$$

This kind of automata and their languages were discovered and studied by Bossut in his PHD [4] without the help of the theory of PROs.

Example 7. In the aim to illustrate the construction, we consider, as in [17], the example of the Bossut's brick wall [4].

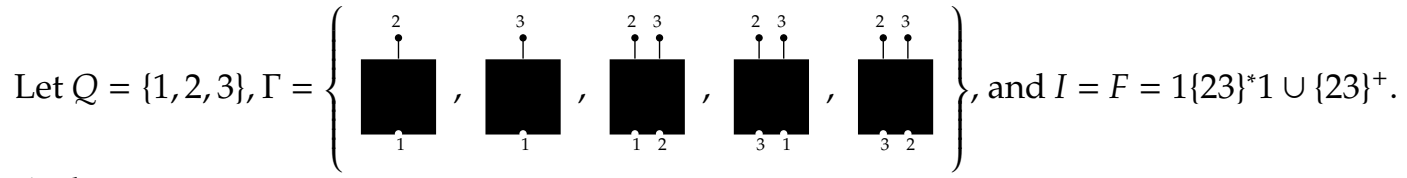

We have

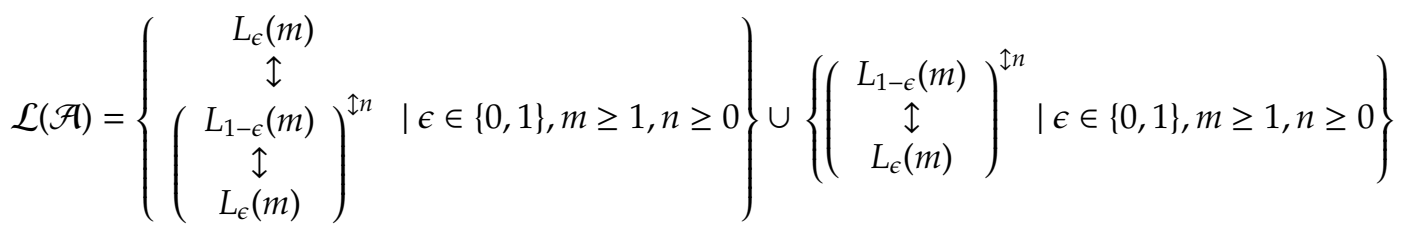

where the $L_{\epsilon}(m)$ are defined recursively by

$$
L_{0}(1)=L_{0}(2 m)=L_{0}(2 m-1) \leftrightarrow \quad, L_{0}(2 m+1)=L_{0}(2 m-1) \leftrightarrow
$$

and

$$
\left.L_{1}(2)=\stackrel{i}{i}, L_{1}(2 m+1)=L_{1}(2 m) \leftrightarrow \stackrel{i}{i}, L_{1}(2(m+1))=L_{0}(2 m)\right) \leftrightarrow
$$


$\uparrow$

$L_{0}(8)$

For instance, the element $\uparrow$ corresponds graphically to the following wall:

$L_{1}(8)$

$\uparrow$

$L_{0}(8)$

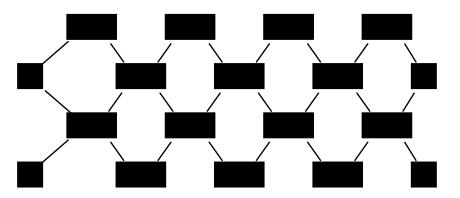

Let us show that such a language can be completely described in terms of multilinear representations. First we consider the free PRO generated by the $u(x)$ for $x \in \Gamma$ and we set $\mathcal{X}:=\{u(x) \mid x \in \mathcal{F} \Gamma\}$. For any words in $w=w_{0} \ldots w_{k} \in\{1, \ldots, N\}$, we define two matrices

$$
\mathrm{IN}_{w}(N)=\mathbf{E}\left(N, 0, k ;[],\left[w_{1}, \ldots, w_{k}\right]\right) \in \mathbb{B}(N, 0, k)
$$

and

$$
\operatorname{OUT}_{w}(N)=\mathbf{E}\left(N, k, 0 ;\left[w_{1}, \ldots, w_{k}\right],[]\right) \in \mathbb{B}(N, k, 0) .
$$

For any $\mathfrak{x} \in \mathcal{X}$, we define the matrix $\mu_{\Gamma}(\mathfrak{x}) \in \mathbb{B}(N, m, n)$ by $\underset{J}{\mu_{\Gamma}(\mathfrak{I})}=1$ if there exists $x \in \Gamma$ such that $\operatorname{In}(x)=J$, $\operatorname{Out}(x)=I$, and $u(x)=x$ and 0 otherwise. The map $\mu$ is extended in a representation of $\mathcal{F}(\mathcal{X})$. As a consequence of Proposition 7 , the language of the automaton is recovered by the formula

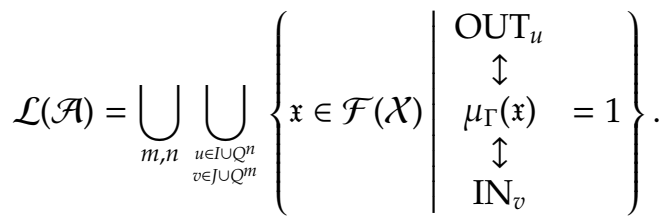

Conversely, to each representation of $\mathcal{X}$, we can associate a unique signature $\Gamma_{\mu}$ such that for each $x \in \Gamma, u(x) \in \mathcal{X}$ and $\underset{\operatorname{Out}(x)}{\mu(x)}(x)=1$.

Definition 5. (Heindel[17]) A language is acceptable if it is the language of a PRO automaton.

As a consequence of formula 45 , we have:

Theorem 4. A language $\mathcal{L}$ is acceptable if and only if there exists a tuple $(Q, \mu, I, J)$ with $Q=\{1, \ldots, N\}$, $I, J \in Q^{*}$, and $\mu$ a representation of $\mathcal{X}$, such that

$$
\left.\mathcal{L}=\bigcup_{m, n} \bigcup_{\substack{u \in \cup \cup Q^{n} \\
v \in J \cup Q^{m}}}\left\{\begin{array}{l|l}
\mathfrak{g} \in \mathcal{F}(\mathcal{X}) & \begin{array}{l}
\mathrm{OUT}_{u} \\
\mathfrak{1} \\
\mu(\mathfrak{g}) \\
\mathfrak{1} \\
\mathrm{IN}_{v}
\end{array}
\end{array}\right\} 1\right\} .
$$

Example 8. The representation associated to the Bossut's wall is defined by

$$
\mu(\square)=\mathbf{E}(3,1,1 ;[2],[1])+\mathbf{E}(3,1,1 ;[3],[1])
$$




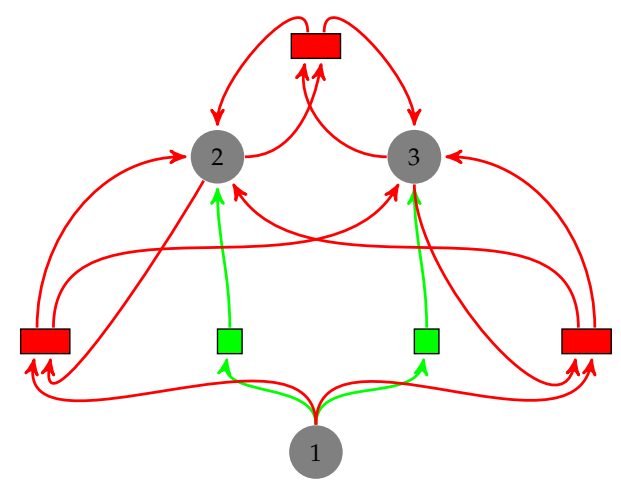

Figure 8: Representation associated to Bossut's wall.

and

$$
\mu(\square)=\mathbf{E}(3,2,2 ;[2,3],[1,2])+\mathbf{E}(3,2,2 ;[2,3],[3,1])+\mathbf{E}(3,2,2 ;[2,3],[3,2])
$$

(see Figure 8).

Let $u=u_{1} \ldots u_{k} \in\{1, \ldots, N\}^{k}$ and $u^{\prime}=u_{1}^{\prime} \ldots u_{k}^{\prime} \in\left\{1, \ldots, N^{\prime}\right\}^{k}$, we define $u \odot v=\left(\left(u_{1}^{\prime}-1\right) M+u_{1}\right) \ldots\left(\left(u_{k}^{\prime}-\right.\right.$ 1) $\left.M+u_{k}\right)$. For instance, if $u=14221 \in\{1, \ldots, 4\}$ and $v=13122 \in\{1, \ldots, 5\}$ then $u \odot v=212265$.

Lemma 7. Let $I$ be a regular language over $Q=\{1, \ldots, N\}$ and $I^{\prime}$ a regular language over $Q^{\prime}=\left\{1, \ldots, N^{\prime}\right\}$ then the language $I \odot I^{\prime}:=\left\{w \odot w^{\prime}\left|w \in I, w^{\prime} \in I^{\prime},\right| w|=| w^{\prime} \mid\right\}$ is regular over $Q^{\prime \prime}=\left\{1, \ldots, N N^{\prime}\right\}$.

Proof. Let $A=(Q, S,\{i\}, F, \delta)$ and $A^{\prime}=\left(Q^{\prime}, S^{\prime},\left\{i^{\prime}\right\}, F^{\prime}, \delta^{\prime}\right)$ be two complete deterministic automata recognizing respectively $I$ and $I^{\prime}$. We consider the automaton $A=\left(Q^{\prime}, S \times S^{\prime},\left\{\left(i, i^{\prime}\right)\right\}, F \times F^{\prime}, \delta^{\prime \prime}\right)$ where $\delta^{\prime \prime}\left(\left(q, q^{\prime}\right),(\beta-1) N+\alpha\right)=\left(p, p^{\prime}\right)$ if and only if $\delta(q, \alpha)=p$ and $\delta^{\prime}\left(q^{\prime}, \alpha\right)=p^{\prime}$. We remark there exists a path from $\left(i, i^{\prime}\right)$ to a final state $\left(f, f^{\prime}\right)$ in $Q^{\prime \prime}$ labeled with $\left(\left(\beta_{1}-1\right) N+\alpha_{1}\right) \cdots\left(\left(\beta_{k}-1\right) N+\alpha_{k}\right)=\alpha_{1} \cdots \alpha_{k} \odot \beta_{1} \cdots \beta_{k}$ if and only if there exists a path from $i$ to $f$ in $Q^{\prime}$ labeled with $\alpha_{1} \cdots \alpha_{k}$ and a path from $i^{\prime}$ to $f^{\prime}$ in $Q^{\prime}$ labeled with $\beta_{1} \cdots \beta_{k}$. We deduce that $I \odot I^{\prime}$ is regular.

Furthermore, from the definition, one obtains

$$
\operatorname{IN}_{u}(N) \odot \operatorname{IN}_{u^{\prime}}\left(N^{\prime}\right)=\operatorname{IN}_{u \odot u^{\prime}}\left(N N^{\prime}\right) \text { and } \operatorname{OUT}_{u}(N) \odot \operatorname{OUT}_{u^{\prime}}\left(N^{\prime}\right)=\operatorname{OUT}_{u \odot u^{\prime}}\left(N N^{\prime}\right) .
$$

Theorem 5. The intersection of two acceptable languages still is acceptable. More precisely, let $\mathcal{L}$ be the language of $A=(\{1, \ldots, N\}, \mu, I, J)$ and $\mathcal{L}^{\prime}$ be the language of $A^{\prime}=\left(\left\{1, \ldots, N^{\prime}\right\}, \mu^{\prime}, I^{\prime}, J^{\prime}\right)$ then

$$
\mathcal{L}\left(\left\{1, \ldots, N N^{\prime}\right\}, \mu \odot \mu^{\prime}, I \odot I^{\prime}, J \odot J^{\prime}\right)=\mathcal{L} \cap \mathcal{L}^{\prime} .
$$

\begin{tabular}{|c|c|c|c|c|c|c|c|c|c|}
\hline $\begin{array}{c}\mathrm{OUT}_{u \odot u^{\prime}} \\
\uparrow\end{array}$ & & $\underset{\uparrow}{\mathrm{OUT}_{u}} \underset{\uparrow}{\odot \mathrm{OUT}_{\mathcal{u}^{\prime}}}$ & & $\mathrm{OUT}_{u}$ & & $\underset{\quad \mathrm{OUT}_{u^{\prime}}}{\stackrel{\imath}{ }}$ & & $\underset{\uparrow}{\mathrm{OUT}_{u}}$ & $\begin{array}{c}\mathrm{OUT}_{u^{\prime}} \\
\quad \downarrow\end{array}$ \\
\hline$\mu \widehat{\odot} \mu^{\prime}(\mathfrak{x})$ & $=$ & $\mu(\mathfrak{x}) \odot \mu^{\prime}(\mathfrak{x})$ & $=$ & $\mu(\mathfrak{x})$ & $\odot$ & $\mu^{\prime}(\mathfrak{x})$ & $=$ & $\mu(\mathfrak{x})$ & $\mu^{\prime}(\mathfrak{X})$ \\
\hline$\uparrow$ & & $\uparrow$ & & $\uparrow$ & & $\uparrow$ & & $\uparrow$ & $\uparrow$ \\
\hline $\mathrm{IN}_{v \odot v^{\prime}}$ & & $\mathrm{IN}_{v} \odot \mathrm{IN}_{v^{\prime}}$ & & $\mathrm{IN}_{v}$ & & $\mathrm{IN}_{v^{\prime}}$ & & $\mathrm{IN}_{v}$ & $\mathrm{IN}_{v^{\prime}}$ \\
\hline
\end{tabular}

Proof. Notice first that Lemma 7 implies that $I \odot I^{\prime}$ and $J \odot J^{\prime}$ are regular.

We remark also that

And so

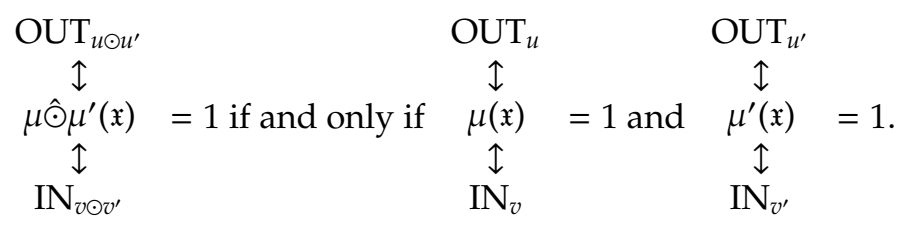


This ends the proof.

Also we have

Theorem 6. (Bossut et al [5]) If $\mathcal{L}$ and $\mathcal{L}^{\prime}$ are acceptable then $\mathcal{L} \cup \mathcal{L}^{\prime}$ is acceptable.

This theorem can also be seen as a consequence of Theorem 3 .

\subsection{A universal definition for automata?}

If there were no question mark, this title would be a bit pretentious. Indeed, it does not really make sense to look for a definition of a universal automaton in an absolute way but rather in relation to a property that we would like to conserve. It is the matrix vision of the automata that we want to keep through the use of representations and a generalized notion of paths. In this quest for a universal definition, we showed two candidates each with their own strengths and weaknesses. The first one comes from sections 6.1, 6.2 and 6.3. In this model, there are no distinction between representations and automata. We are therefore immersed in the heart of the algebraic tools and this gives coherence to this model. For instance, there are no more initial or final states, nor more generally, input and output vectors. All states play a similar role and the automaton produces a number when we give it an element with neither input nor output. Although it is algebraically interesting, this model does not give a general definition of recognizable languages, but a multitude of different theories of recognizability depending on the question we want to ask and on the form of the elements of the PRO. The second candidate is due to Bossut [4, 5] (while the connexion with PROs was pointed out by Heindel [17]) and was studied in Section 6.4. This model may seem a little less elegant than the first one (although it is a matter of point of view) because it forces us to manipulate a triplet instead of only one representation. Indeed, together with the representation, we need two sets $I$ and $J$ to manage the inputs and outputs of the automata. Nevertheless, it is very well adapted to the theory of languages because it allows us to capture a general notion of recognizability (says acceptability in [17]).

Notice also that the two models can be extends to manipulate commutative multiplicities. For the first one, it is straightforward since it suffices to replace the boolean semiring by the suitable commutative semiring. For the second model, we need besides to replace the regular languages $I$ and $J$ by regular series. Hence, we define the series of an automaton by

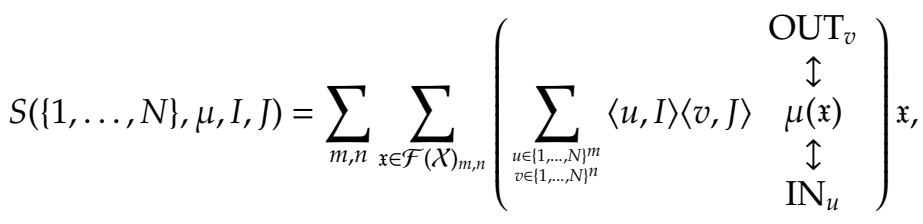

where $\langle w, I\rangle$ denotes the coefficient of the word $w$ in the series $I$.

\section{Conclusion and perspectives}

Throughout the paper we have investigated several properties of the multilinear representations of free PROs. In a way, this validates the diagrammatic approaches for multi-linear calculations. As an illustration, we describe in Appendix Bhow quantum circuits can be seen as representations of a free PRO. The cases of circuits Pros is easier to understand because the combinatorial description is well known. For more general PROs, the combinatorial description is less easy and has been less studied. It seems to be based on isotopic classes of some graphs. However, such representations appear naturally in the literature (see Section[C for the example of Temperley-Lieb algebras). Any advances in the understanding of the combinatorial aspect of free Pros should have many applications. For instance, it should be interesting to understand which notion extends the connectivity in the aim to generalize 
Theorem 3 ,

In the last section, we saw that free PROs procide an ideal framework for defining a general notion of automata embracing most of the classical definitions (word automata, tree automata, branching automata) and based on (multi)-linear algebra. Nevertheless, word automata with non-commutative multiplicities do not fit into that pattern. Indeed, when $\mathbb{K}$ is not commutative, $\mathbb{K}(N)$ is not a PRO because the intechange law no longer holds. In the same way, it is not possible to simulate the behavior of transducers with the proposed constructions. All of these remarks suggest that one has to define more general multiplicities having algebraic structure close than those of PROs, for instance by choosing a convenient structure of enriched PRO.

Another direction of research will consist in investigating generalizations of the Kleene-Schutzenberger theorem for autom for PRO-automata and characterizing regularseries by introducing kinds of rational operations playing the same role as the catenation and the Kleene star for regular (word) languages. Notice that a Kleene theorem for Bossut automata was proved in [5]. In this paper it is proved that a language $\mathcal{L}$ is accepted by an automaton if and only if it can be described using the symbols together with 5 rational operations: vertical composition, vertical star, horizontal composition, horizontal star, and union. It should be interesting to investigate the hypermatrix counter-parts of these operations and how they can be generalized to series.

Acknowledgment This work was partially supported by ERDF project MOUSTIC.

\section{References}

[1] N. Bedon, Logic and branching automata,Proceedings of MFCS 2013: 38nd International Symposium on Mathematical Foundations of Computer Science, Klosterneuburg, Austria, Krishnendu Chatterjee and Jiri Sgall Eds, vol. 8087 of Lecture Notes in Computer Science, pp 123-134, 2013

[2] J. M. Boardman, R. M. Vogt, Homotopy-everything H-spaces , Bull. Amer. Math. Soc. 74 1117-1122 (1968).

[3] J. M.Boardman, R. M. Vogt, Homotopy Invariant Algebraic Structures on Topological Spaces. Lecture Notes in Mathematics 347, Springer-Verlag (1973).

[4] F. Bossut, Rationalité et reconnaissabilité dans les graphes, PhD thesis, UniversitÃ̂l' Lille, Sciences et Technologies, 1986.

[5] F. Bossut, M. Dauchet, and B. Warin. A Kleene theorem for a class of planar acyclic graphs, Information and Computation, 117(2):251-265, 1995.

[6] J.-P. Bultel, S. Giraudo, Combinatorial Hopfalgebras from PROs, Journal of Algebraic Combinatorics 44 (2) 455-493, 2016.

[7] B. Coecke, É.O. Paquette, Categories for the practising physicist, in New Structures for Physics, Volume 813, Lecture Notes in Physics, p 173-286 Springer-Verlag, 2010.

[8] N. Chomsky, Three models for the description of language, Transactions on Information Theory 2 (1956), 113-124.

[9] N. Chomsky, and M.P. Schützenberger, The algebraic theory of context free languages, In Braffort, P. Hirschberg, D. Computer Programming and Formal Languages. Amsterdam: North Holland (1963), 118-161.

[10] F. Drewes, Lecture notes on Tree Automata, Frank Drewes Ed., Umea University. 
[11] B. Eckmann, P. J. Hilton, Group-like structures in general categories. I. Multiplications and comultiplications, Mathematische Annalen 145 (3): 227âĂŞ255, 1962.

[12] S. Eilenberg, Automata, languages, and machines, Pure and Applied Mathematics v 59A, Academic press, 1974.

[13] A. Ekert, Introduction to quantum computation, in Fundamentals of quantum informations, Dieter Heiss (ed.), Springer-Verlag 2002.

[14] P. Etingof, S. Gelaki, D. Nikshych, and V. Ostrich, Tensor Categories, Mathematical Survey and Monographs, volume 205, American Mathematical Society, 2010.

[15] Z. Fülöp, H. Vogler, Weighted Tree Automata and Tree transducers, in Handbook of Weighted automata, Droste-Kuich-Vogler (Eds), Springer 2009.

[16] J.S. Golan, Semirings and their applications, Springer Netherlands, 1999.

[17] T. Heindel, A Myhill-Nerode Theorem beyond Trees and Forests via Finite Syntactic Categories Internal to Monoids, unpublished http://www.informatik.uni-leipzig.de/ heindel/MyhillNerodeFoSSaCS18.pdf

[18] S.C. Kleene,Representation of events in nerve nets and finite automata, In: Shannon, C.E., McCarthy, J. (eds.), Automata Studies, Princeton University Press, Princeton (1956), 3-41.

[19] A. Joyal, R. Street, Planar diagrams and tensor algebra, manuscript http://maths.mq. edu.au/ street/PlanarDiags.pdf, 1988.

[20] T. Leinster, Higher Operads, Higher Categories . London Math. Soc. Lecture Note Series. Cambridge University Press, Cambridge, 2004.

[21] K. Lodaya and P. Weil, A Kleene iteration for parallelism, In Foundations of Software Technology and Theoretical Computer Science, pages 355-366, 1998.

[22] K. Lodaya and P. Weil, Series-parallel languages and the bounded-width property, Theoret. Comput. Sci. , 237(1-2):347-380, 2000

[23] S. Mac Lane; Categorical algebra, Bull. Amer. Math. Soc. 71 40-106 (1965).

[24] S. Mac Lane, Natural associativity and commutativity, Rice Univ. Studies 49 28-46 (1963).

[25] W.S., McCulloch and E. Pitt, A logical calculus of the ideas immanent in nervous activity, Bulletin of Mathematical Biophysics (1943) 541-544.

[26] M.O. Rabin and D. Scott, Finite Automata and Their Decision Problems, IBM Journal of Research and Development 3 (2) (1959) 114-125.

[27] M. P. Schützenberger, On the definition of a family of automata, Inf. Control 4 (1961), 245-270.

[28] M. Takahashi, On the bordism categories III, Math. Sem. Notes Kobe Univ. 10 (1982), 211-236.

[29] N. Temperley, E. Lieb, Relations between the 'Percolation' and 'Colouring' Problem and other GraphTheoretical Problems Associated with Regular Planar Lattices: Some Exact Results for the 'Percolation' Problem, Proceedings of the Royal Society Series A 322 (1971), 251-280. 


\section{A ModPro and enriched categories}

Let $(\mathcal{M}, \otimes, \alpha, \lambda, \rho, I)$ be a monoidal category. A $\mathcal{M}$-category C (also called enriched category) consists of

- the class $\operatorname{Obj}(\mathrm{C})$ of the objects of $\mathrm{C}$,

- an object $\mathrm{C}(\mathrm{a}, \mathrm{b})$ of $\mathcal{M}$ for any pair of objects $(\mathrm{a}, \mathrm{b})$ of $\mathrm{C}$,

- for each object a in $\mathrm{C}$, the identity $I d_{\mathrm{a}}$ is an arrow $I \rightarrow \mathrm{C}(\mathrm{a}, \mathrm{a})$ in $\mathcal{M}$, where $I$ denotes the identity object in $\mathcal{M}$,

- the composition of the arrows in $\mathrm{C}$ is encoded by the tensor product in $\mathrm{M}$. More precisely, we have for each triple $(a, b, c)$ of objects in $C$ a composition $o_{a b c}: C(b, c) \otimes C(a, b) \rightarrow C(a, c)$, which is an arrow in $\mathcal{M}$, such that

- For any a, b, c, din C, we have

$$
\mathrm{o}_{\mathrm{abd}}\left(\mathrm{o}_{\mathrm{bcd}} \otimes 1\right)=\mathrm{o}_{\mathrm{acd}}\left(1 \otimes \mathrm{o}_{\mathrm{abc}}\right) \alpha:(\mathrm{C}(\mathrm{c}, \mathrm{d}) \otimes \mathrm{C}(\mathrm{b}, \mathrm{c})) \otimes \mathrm{C}(\mathrm{a}, \mathrm{b}) \rightarrow \mathrm{C}(\mathrm{a}, \mathrm{d}),
$$

- For any pair of objects $(a, b)$ in $C$, we have

$$
\mathrm{o}_{\mathrm{abb}}\left(I d_{\mathrm{b}} \otimes 1\right)=\lambda: I \otimes \mathrm{C}(\mathrm{a}, \mathrm{b}) \rightarrow \mathrm{C}(\mathrm{a}, \mathrm{b}),
$$

- For any pair of objects $(a, b)$ in $C$, we have

$$
\mathbf{o}_{\mathrm{aab}}\left(1 \otimes I d_{\mathrm{a}}\right)=\rho: \mathrm{C}(\mathrm{a}, \mathrm{b}) \otimes I \rightarrow \mathrm{C}(\mathrm{a}, \mathrm{b}) .
$$

Example 9. Ordinary categories are categories enriched over Set the category of sets with cartesian product.

Example 10. Consider the strict monoidal category 2 whose objects are $\{0,1\}$ (1 is the identity) with a single nonidentity arrow $0 \rightarrow 1$ and such that the cartesian product is the ordinary product of integers. Each preordered sets $\mathcal{P}$ can be seen as a category enriched over 2 . Notice that $\mathcal{P}(a, b)=1$ means that $a \leq b$ and the composition encodes the transitivity. Indeed, if $\mathcal{P}(a, b)=1$ and $\mathcal{P}(b, c)=1$ then necessarily we have $\mathcal{P}(a, c)=1$ because there is no arrow $1 \rightarrow 0$ in 2 .

The notions of enriched functors and enriched natural transformations are defined as for categories. More precisely, if $\mathrm{C}$ and $\mathrm{D}$ are two $\mathcal{M}$-categories, a $\mathcal{M}$-functor $\mathrm{F}: \mathrm{C} \rightarrow \mathrm{D}$ assigns to each object a of $\mathrm{C}$ an object $F(a)$ in $D$. It also associates with each arrow $C(a, b)$ a morphism $F(a, b): C(a, b) \rightarrow D(F(a), F(b))$ in $\mathcal{M}$ such that $\mathrm{F}(\mathrm{a}, \mathrm{a}) I d_{\mathrm{a}}=I d_{\mathrm{F}(\mathrm{a})}: I \rightarrow \mathrm{D}(\mathrm{F}(\mathrm{a}), \mathrm{F}(\mathrm{a}))$ and $\mathrm{F}(\mathrm{a}, \mathrm{c}) \mathrm{o}=\mathrm{oF}(\mathrm{b}, \mathrm{c}) \otimes \mathrm{F}(\mathrm{a}, \mathrm{b}): \mathrm{C}(\mathrm{b}, \mathrm{c}) \otimes \mathrm{C}(\mathrm{a}, \mathrm{b}) \otimes \mathrm{D}(\mathrm{F}(\mathrm{a}), \mathrm{F}(\mathrm{b}))$.

Let $C$ and $D$ be two enriched categories and F, G : C $\rightarrow$ D be two enriched functors. An enriched natural transformation $\boldsymbol{\eta}: \mathrm{F} \rightarrow \mathrm{G}$ is a family of morphisms $\eta_{\mathrm{a}}: I \rightarrow \mathrm{D}(\mathrm{F}(\mathrm{a}), \mathrm{G}(\mathrm{a})$ ) of morphism of $\mathcal{M}$ defined for each object $\mathrm{a}$ in $\mathrm{C}$ such that for any two objects $\mathrm{a}$ and $\mathrm{b}$ of $\mathrm{C}$ we have $o_{D}\left(G(a, b) \otimes \eta_{\mathrm{a}}\right) \rho^{-1}=$ $o_{D}\left(\eta_{b} \otimes F(a, b)\right) \lambda^{-1}: C(a, b) \rightarrow D(F(a), G(b))$.

Recall that a monoidal category $\mathcal{M}$ is symmetric if there exists a natural isomorphism com such that for any object $a, b \in \mathcal{M}$, com $: a \otimes b \simeq b \otimes a$ satisfying

- The first hexagonal identity. $\alpha \operatorname{com} \alpha=(1 \otimes \operatorname{com}) \alpha(\operatorname{com} \otimes 1)$,

- Self inversibility. com com $=1$.

Notice that these two identities imply the second hexagonal identity which reads $\alpha^{-1}$ com $\alpha^{-1}=$ $(\operatorname{com} \otimes 1) \alpha^{-1}(1 \otimes \operatorname{com})$. Let $(\mathcal{M}, \otimes, \alpha, \lambda, \rho, I, \mathrm{com})$ be a symmetric monoidal category and $\mathrm{C}$ be a $\mathcal{M}-$ category. We consider the natural transformation $\phi$ defined by $\phi:=\alpha^{-1}(1 \otimes \alpha)(1 \otimes(\operatorname{com} \otimes 1))\left(1 \otimes \alpha^{-1}\right) \alpha$. We denote $\mathrm{C} \times \mathrm{D}$ the structure consisting of 
- a class $\operatorname{Obj}(C \times D)$ of objects of $C \times D$ which are the pairs $[a, b]$ of objects,

- the arrows are objects $(C \times D)\left(\left[a_{1}, b_{1}\right],\left[a_{2}, b_{2}\right]\right)=C\left(a_{1}, a_{2}\right) \otimes D\left(b_{1}, b_{2}\right)$ of $\mathcal{M}$ such that $a_{1}, a_{2}$ are objects in $C$ (resp. $b_{1}, b_{2}$ are objects in $\left.D\right)$ and $C\left(a_{1}, a_{2}\right)$ (resp. $\left.D\left(b_{1}, b_{2}\right)\right)$ are arrows in $C$ (resp. D),

- For each $O_{1}=\left[\mathrm{a}_{1}, \mathrm{~b}_{1}\right], O_{2}=\left[\mathrm{a}_{2}, \mathrm{~b}_{2}\right], O_{3}=\left[\mathrm{a}_{3}, \mathrm{~b}_{3}\right]$ objects in $\mathrm{C} \times \mathrm{D}$, we define the arrow $\circ_{\mathrm{O}_{1}, \mathrm{O}_{2}, \mathrm{O}_{3}}$ : $(\mathrm{C} \times \mathrm{D})\left[\mathrm{O}_{2}, \mathrm{O}_{3}\right] \otimes(\mathrm{C} \times \mathrm{D})\left[\mathrm{O}_{1}, \mathrm{O}_{2}\right] \longrightarrow(\mathrm{C} \times \mathrm{D})\left[\mathrm{O}_{1}, \mathrm{O}_{3}\right]$ in $\mathcal{M}$ designating a composition and defined by $o_{O_{1}, O_{2}, O_{3}}=\left(o_{a_{1}, a_{2}, a_{3}} \otimes o_{b_{1}, b_{2}, b_{3}}\right) \phi$. Notice that $\phi$ is the isomorphism

$$
\left(C\left(a_{2}, a_{3}\right) \otimes D\left(b_{2}, b_{3}\right)\right) \otimes\left(C\left(a_{1}, a_{2}\right) \otimes D\left(b_{1}, b_{2}\right)\right) \longrightarrow\left(C\left(a_{2}, a_{3}\right) \otimes C\left(a_{1}, a_{2}\right)\right) \otimes\left(D\left(b_{2}, b_{3}\right) \otimes D\left(b_{1}, b_{2}\right)\right) .
$$

Proposition 8. If $\mathrm{C}$ and $\mathrm{D}$ are $\mathcal{M}$-categories then $\mathrm{C} \times \mathrm{D}$ is a $\mathcal{M}$-category

Proof. Let $O_{i}=\left[\mathrm{a}_{i}, \mathrm{~b}_{i}\right], i=1 \ldots 4$, be four objets of $\mathrm{C} \times \mathrm{D}$. Consider the map

$$
\mathrm{o}_{\mathrm{O}_{1}, \mathrm{O}_{2}, \mathrm{O}_{4}}\left(\mathrm{o}_{\mathrm{O}_{2}, \mathrm{O}_{3}, \mathrm{O}_{4}} \otimes 1\right):\left((\mathrm{C} \times \mathrm{D})\left(\mathrm{O}_{3}, \mathrm{O}_{4}\right) \otimes(\mathrm{C} \times \mathrm{D})\left(\mathrm{O}_{2}, \mathrm{O}_{3}\right)\right) \otimes(\mathrm{C} \times \mathrm{D})\left(\mathrm{O}_{1}, \mathrm{O}_{2}\right) \longrightarrow(\mathrm{C} \times \mathrm{D})\left(\mathrm{O}_{1}, \mathrm{O}_{4}\right) .
$$

We have

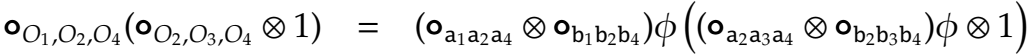

$$
\begin{aligned}
& =\left(\left(o_{a_{1} a_{2} a_{4}}\left(o_{a_{2} a_{3} a_{4}} \otimes 1\right)\right) \otimes\left(o_{b_{1} b_{2} b_{4}}\left(o_{b_{2} b_{3} b_{4}} \otimes 1\right)\right)\right) \phi(\phi \otimes 1) .
\end{aligned}
$$

But, since $\mathcal{M}$ is monoidal one has

$$
\mathrm{o}_{\mathrm{a}_{1} \mathrm{a}_{2} \mathrm{a}_{4}}\left(\mathrm{o}_{\mathrm{a}_{2} \mathrm{a}_{3} \mathrm{a}_{4}} \otimes 1\right)=\mathrm{o}_{\mathrm{a}_{1} \mathrm{a}_{3} \mathrm{a}_{4}}\left(1 \otimes \mathrm{o}_{\mathrm{a}_{1} \mathrm{a}_{2} \mathrm{a}_{3}}\right) \alpha \text { and } \mathrm{o}_{\mathrm{b}_{1} \mathrm{~b}_{2} \mathrm{~b}_{4}}\left(\mathrm{o}_{\mathrm{b}_{2} \mathrm{~b}_{3} \mathrm{~b}_{4}} \otimes 1\right)=\mathrm{o}_{\mathrm{b}_{1} \mathrm{~b}_{3} \mathrm{~b}_{4}}\left(1 \otimes \mathrm{o}_{b_{1} b_{2} \mathrm{~b}_{3}}\right) \alpha .
$$

Noting that

$$
(\alpha \otimes \alpha) \phi(\phi \otimes 1)=\phi(1 \otimes \phi) \alpha,
$$

from

$$
\left(\left(o_{a_{1}, a_{3}, a_{4}}\left(1 \otimes o_{a_{1}, a_{2}, a_{3}}\right)\right) \otimes\left(o_{b_{1}, b_{3}, b_{4}}\left(1 \otimes o_{b_{1}, b_{2}, b_{3}}\right)\right) \phi=\left(o_{a_{1}, a_{3}, a_{4}} \otimes o_{b_{1} b_{3} b_{4}}\right) \phi\left(1 \otimes\left(o_{a_{1} a_{2} a_{3}} \otimes o_{b_{1} b_{2} b_{3}}\right)\right),\right.
$$

we deduce

$$
\mathbf{o}_{\mathrm{O}_{1} \mathrm{O}_{2} \mathrm{O}_{4}}\left(\mathbf{o}_{\mathrm{O}_{2} \mathrm{O}_{3} \mathrm{O}_{4}} \otimes 1\right)=\mathbf{o}_{\mathrm{O}_{1} \mathrm{O}_{3} \mathrm{O}_{4}}\left(1 \otimes \mathbf{o}_{\mathrm{O}_{1} \mathrm{O}_{2} \mathrm{O}_{3}}\right) \alpha .
$$

Furthermore, since $I d_{O_{i}}=I d_{\mathrm{a}_{i}} \otimes I d_{\mathrm{b}_{i}}$, we have

$$
\begin{aligned}
\mathbf{o}_{\mathrm{O}_{1} \mathrm{O}_{2} \mathrm{O}_{2}}\left(I d_{\mathrm{O}_{2}} \otimes 1\right) & =\left(\mathrm{o}_{\mathrm{a}_{1} \mathrm{a}_{2} \mathrm{a}_{2}} \otimes \mathbf{o}_{\mathrm{b}_{1} \mathrm{~b}_{2} \mathrm{~b}_{2}}\right)\left(\left(I d_{\mathrm{a}_{2}} \otimes 1\right) \otimes\left(I d_{\mathrm{b}_{2}} \otimes 1\right)\right) \phi \alpha^{-1} \lambda^{-1} \\
& =(\lambda \otimes \lambda) \phi \alpha^{-1} \lambda^{-1}=\lambda
\end{aligned}
$$

and

$$
\begin{aligned}
& { }^{\circ}{ }_{\mathrm{O}_{1} \mathrm{O}_{1} \mathrm{O}_{2}}\left(1 \otimes I d_{\mathrm{O}_{1}}\right)=\left(\mathrm{o}_{\mathrm{a}_{1} \mathrm{a}_{1} \mathrm{a}_{2}} \otimes \mathrm{o}_{\mathrm{b}_{1} \mathrm{~b}_{1} \mathrm{~b}_{2}}\right)\left(\left(1 \otimes I d_{\mathrm{a}_{1}}\right) \otimes\left(1 \otimes I d_{\mathrm{b}_{1}}\right)\right) \phi \rho^{-1} \lambda^{-1} \\
& =(\rho \otimes \rho) \phi \alpha^{-1} \rho^{-1}=\rho \text {. }
\end{aligned}
$$

Equations (47), (48) and (49) show that $\mathrm{C} \times \mathrm{D}$ is an enriched category.

An enriched $\mathcal{M}$-monoidal category $\mathrm{M}$ is a $\mathcal{M}$-category equiped with a bifunctor $\otimes: \mathrm{M} \times \mathrm{M} \rightarrow \mathrm{M}$, an object I called the unit object and three natural $\mathcal{M}$-isomorphisms

1. the associator $\boldsymbol{\alpha}$ with components $\boldsymbol{\alpha}_{\mathrm{a}, \mathrm{b}, \mathrm{c}}:(\mathrm{a} \otimes \mathrm{b}) \otimes \mathrm{c} \simeq \mathrm{a} \otimes(\mathrm{b} \otimes \mathrm{c})$,

2. the left unitor $\lambda$ with components $\lambda_{\mathrm{a}}: I \otimes \mathrm{a} \simeq \mathrm{a}$,

3. the right unitor $\rho$ with components $\rho_{\mathrm{a}}: \mathrm{a} \otimes I \simeq \mathrm{a}$ 
satisfying $(1 \otimes \alpha) \alpha(\alpha \otimes 1)=\alpha \boldsymbol{\alpha}:((\mathrm{a} \otimes \mathrm{b}) \otimes \mathrm{c}) \otimes \mathrm{d} \rightarrow \mathrm{a} \otimes(\mathrm{b} \otimes(\mathrm{c} \otimes \mathrm{d}))$ and $(1 \otimes \lambda) \boldsymbol{\alpha}=\rho \otimes 1:(\mathrm{a} \otimes I) \otimes \mathrm{b} \rightarrow \mathrm{a} \otimes \mathrm{b}$ for any objects $\mathrm{a}, \mathrm{b}, \mathrm{c}, \mathrm{d}$ in $\mathrm{M}$. A enriched $\mathcal{M}$-monoidal category is said strict if the natural transformations $\boldsymbol{\alpha}, \boldsymbol{\lambda}, \boldsymbol{\rho}$ are identities. Hence, we can now define the notion of enriched PRO as a strict $\mathcal{M}$-monoidal category whose objects are the natural numbers and the tensor product sends $(m, n)$ to $m+n$.

Let $\mathbb{K}$ be a commutative semiring. The class of $\mathbb{K}$-modules forms a category which is equipped with a tensor product conferring to it a structure of symmetric monoidal category. For any $\mathbb{K}$-module $M$, the identity object is $\mathbb{K}$. The morphism $\lambda_{M}$ (resp. $\left.\rho_{M}\right)$ sends $(a, m)(\operatorname{resp} .(m, a))$ to $a \cdot m$. If $M, N$, and $P$ are three $\mathbb{K}$-modules, the morphism $\alpha_{M, N, P}$ sends $((m, n), p)$ to $(m,(n, p))$.

Definition 6. A K-ModPro is a PRO enriched in the category of the $\mathbb{K}$-modules.

Notice that the compositions and the tensor product are morphisms of $\mathbb{K}$-modules.

This definition is equivalent to the definition given in Section 2.4

- The operation $\leftrightarrow$ is assimilated to the bifunctor $\otimes$. Hence, it sends $(\mathrm{p}, \mathrm{q}+\mathrm{r}) \in \operatorname{Hom}(m, n) \otimes$ $\operatorname{Hom}\left(m^{\prime}, n^{\prime}\right)$ to $\mathrm{p} \leftrightarrow(\mathrm{q}+\mathrm{r}) \in \operatorname{Hom}\left(m+m^{\prime}, n+n^{\prime}\right)$ and $(\mathrm{p}, \mathrm{q})+(\mathrm{p}, \mathrm{r})$ to $(\mathrm{p} \leftrightarrow \mathrm{q})+(\mathrm{p} \leftrightarrow \mathrm{r})$. Since $(\mathrm{p}, \mathrm{q}+\mathrm{r})=(\mathrm{p}, \mathrm{q})+(\mathrm{p}, \mathrm{r})$, we deduce that $\mathrm{p} \leftrightarrow(\mathrm{q}+\mathrm{r})=(\mathrm{p} \leftrightarrow \mathrm{q})+(\mathrm{p} \leftrightarrow \mathrm{r})$. In the same way, the right distributivity is deduced from the fact that $\leftrightarrow=\otimes$.

- The operation $\mathfrak{l}$ is assimilated to the composition $\bullet$. Hence, it sends $(\mathrm{p}, \mathrm{q}+\mathrm{r}) \in \operatorname{Hom}(m, n) \otimes$

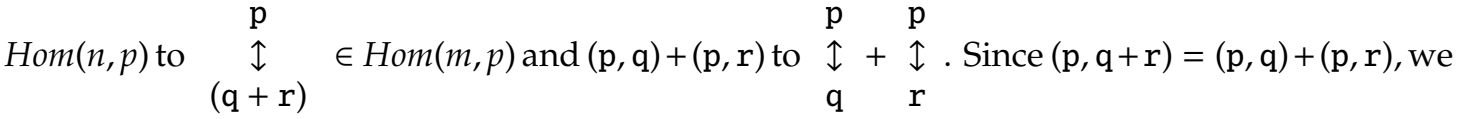

$$
\begin{aligned}
& \text { deduce that } \begin{array}{c}
\left(\mathrm{p}_{1}+\mathrm{p}_{2}\right) \\
\mathfrak{\downarrow} \\
\mathrm{q}
\end{array}=\left(\begin{array}{c}
\mathrm{p}_{1} \\
\uparrow \\
\mathrm{q}
\end{array}\right)+\left(\begin{array}{c}
\mathrm{p}_{2} \\
\uparrow \\
\mathrm{q}
\end{array}\right), \begin{array}{c}
\mathrm{q} \\
\left(\mathrm{p}_{1}+\mathrm{p}_{2}\right)
\end{array} \text {. In the same way, the down distributivity }
\end{aligned}
$$
is deduced from the fact that $\uparrow=0$.

The following table summarize the correspondance between the categorial definition and the constructive definition.

\begin{tabular}{|c|c|}
\hline Constructive definition & Categorial definition \\
\hline $\mathrm{M}_{m, n}$ & $\mathrm{M}(m, n)$ \\
$\uparrow$ & $\circ$ \\
$\leftrightarrow$ & $\otimes$ \\
\hline
\end{tabular}

\section{B Modeling quantum gates}

Representation of free PRos can be used when we draw some multilinear operations as boxes. For instance, the composition of quantum gates, in quantum computation theory, can be represented by hypermatrices in $\mathbb{C}(2)$. Quantum networks are devices consisting of quantum logic gates whose computational steps are synchronized in times (see eg [13]). Graphically a quantum networks is drawn by relying quantum gates. Quantum gates are identified with hypermatrices of $\mathbb{C}(2)_{n, n}$ acting on qubit systems identified with hypermatrices of $\mathbb{C}(2)_{0, m}$. In the aim to match with the notation of quantum computation theory, we consider that the indices of the hypermatrices are taken in $\{0,1\}$ instead of $\{1,2\}$. The most common gate is the Hadamard gate $H$ acting on a single qubit. This gate is identified with the matrix $\mathcal{H} \in \mathbb{C}(2)_{1,1}$ defined by $\underset{0}{\mathcal{H}}=\underset{1}{\stackrel{\mathcal{H}}{\mathcal{H}}}=\underset{0}{\stackrel{\mathcal{H}}{H}}=-\underset{1}{\mathcal{H}}=\frac{1}{\sqrt{2}}$. Another well known gate is denoted by $c-V$ acting on two qubit systems as the hypermatrix $\mathcal{V}$ in $\mathbb{C}(2)_{2,2}$ defined by $\stackrel{a b}{V}=1$ for any $(a, b) \neq(1,1), \stackrel{11}{\mathcal{V}}=I$, and $\underset{c d}{\stackrel{a b}{V}}=0$ for $(a, b) \neq(c, d)$. Some important gates can be obtained by sticking these elementary gates in quantum networks. 
Let us give a few examples. The controlled-not gate $C$ is one of the most popular two-qubit gates because it allows to create entanglement from non entangled systems. Graphically, the network is drawn in Figure 9. Indeed, all works as if we consider the free Pro generated by $H$ and $c-V$

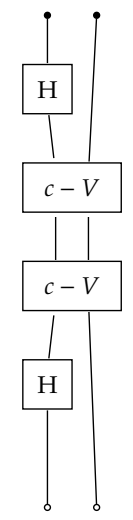

Figure 9: An example of Quantum Network.

together with the representation $\mu$ sending $H$ to $\mathcal{H}$ and $c-V$ to $\mathcal{V}$. The hypermatrix $C=\mu(C)$ can

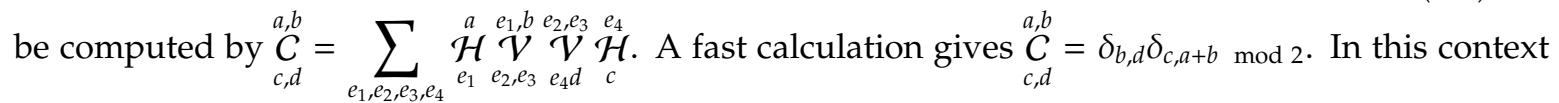
a (pure) $k$-qubit system $|\phi\rangle=\sum_{i_{1}, \ldots, i_{k}} \alpha_{i_{1}, \ldots, i_{k}}\left|i_{1} \cdots i_{k}\right\rangle$ is seen as the hypermatrix $\phi \in \mathbb{C}(2)_{0, k}$ satisfying $\underset{i_{1}, \ldots, i_{k}}{\phi}=\alpha_{i_{1}, \ldots, i_{k}}$. The action of a quantum network $N$ on a system $|\phi\rangle$ is just the branching $\begin{gathered}\phi \\ \mathcal{I}\end{gathered}$, where $\mathcal{N}$ is the hypermatrix associated to $N$. For instance, let $|\phi\rangle=|01\rangle+|11\rangle$. The matrix $\begin{aligned} & \phi \\ & \stackrel{C}{C}\end{aligned}$ is associated to the quantum system $\left|\phi^{\prime}\right\rangle=|00\rangle+|11\rangle$. Notice that $\left|\phi^{\prime}\right\rangle$ is entangled but not $|\phi\rangle$.

\section{On Temperley-Lieb algebras}

We consider the graded set $X=\mathcal{X}_{2,0} \cup \mathcal{X}_{0,2}$ with $\mathcal{X}_{2,0}=\{\cup\}$ and $X_{0,2}=\{\cap\}$.

We define the diagram ModPro $\mathcal{D}=\left.\mathbb{C}\langle X\rangle\right|_{\equiv_{D}}$ where $\equiv_{D}$ is the congruence generated by

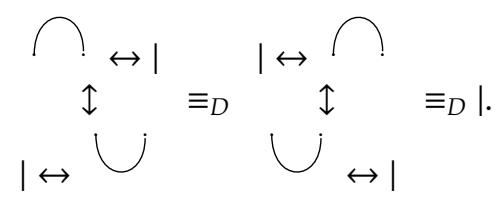

Let

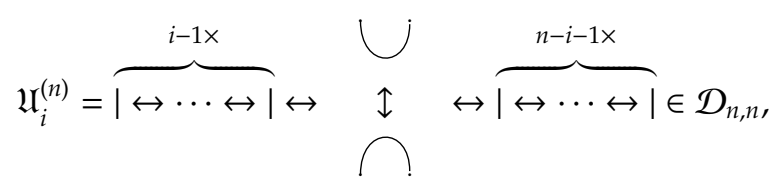


for $1 \leq i<n$. These elements satisfy the commutations $\mathfrak{r}_{i}^{(n)} \mathfrak{d}_{j}^{(n)}=\mathfrak{l}_{j}^{(n)} \mathfrak{d}_{i}^{(n)}$ for $|i-j|>1$ together with reduction of braids

$$
\begin{aligned}
& \mathfrak{r}_{i}^{(n)} \\
& \stackrel{i}{i} \\
& \mathfrak{l}_{i+1}^{(n)} \\
& \mathfrak{i} \\
& \mathfrak{r}_{i}^{(n)}
\end{aligned}=\mathfrak{r}_{i}^{(n)}
$$

and

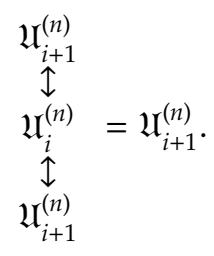

We denote by $\mathcal{T}=\mathcal{D} / \equiv_{T}$, the quotient of $\mathcal{D}$ by the congruence generated by

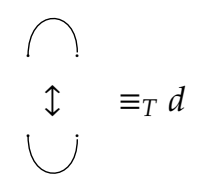

for some $d \in \mathbb{C}$. If we denote $\hat{\mathfrak{U}}_{i}^{(n-1)}=\phi_{\equiv_{T}}\left(\mathfrak{U}_{i}^{(n-1)}\right)$, then one has an additional relation

$$
\underset{\hat{\mathfrak{V}}_{i}^{(n-1)}}{\stackrel{\mathfrak{I}}{(n-1)}}=d \hat{\mathfrak{U}}_{i}^{(n-1)} .
$$

Notice that $\left(T_{n, n}, \mathfrak{\uparrow},+\right)$ is an algebra and the subsalgebra $\mathcal{T} \ell_{n}$ generated by the elements $\hat{\mathfrak{U}}_{i}^{(n-1)}$ is isomorphic to the Temperley-Lieb algebra (see e.g. [29]). As a consequence, any multilinear representation of $\mathcal{T}$ of dimension $N$ gives a representation of $\mathcal{T} \ell_{n}$ of dimension $N^{n}$.

Hence to find a representation of the Temperley-Lieb algebra, it suffices to exhibit a representation $\mu$ of $\mathcal{F}(\mathcal{X})$ such that

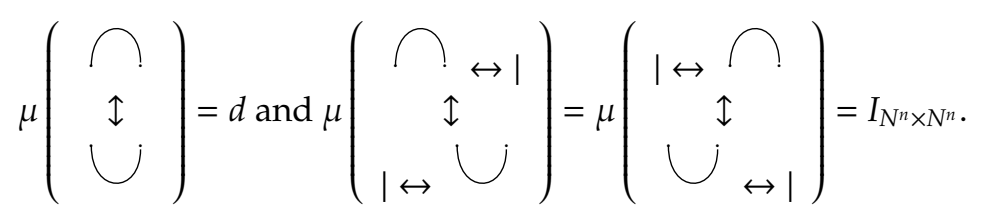

For instance, one can deduce a representation of dimension $2^{n}$ of each Temperley-Lieb algebra $\mathcal{T} \ell_{n}$ from the dimension 2 representation $\mu$ of $\mathcal{F}(\mathcal{X})$ defined by

$$
\mu(\cap)=\left(\begin{array}{cccc}
11 & 12 & 21 & 22 \\
2-d & 0 & d-2 & 1
\end{array}\right)
$$

and

$$
\mu(\bigcup)=\begin{aligned}
& 11 \\
& 12 \\
& 21 \\
& 22
\end{aligned}\left(\begin{array}{c}
\frac{1}{2-d} \\
0 \\
1 \\
1
\end{array}\right)
$$

Let $\mathfrak{D}$ be a diagram in $\mathcal{D}_{n, n}$, we denote by Cycle(D) the design obtained from $\mathfrak{D}$ by relying the $i$ th input to the $i$ th output for each $1 \leq i \leq n$. We remark that Cycle(D) is the juxtaposition of connected 
components. Some components contain at least two generators of $\mathcal{X}$, we will say that such a component is non trivial. The trivial components come from a single $\mid$ in $\mathfrak{D}$. We denote by ntriv(D) (resp. triv(D)) the number of non trivial (resp. odd) connected components in Cycle(D) number of occurrences of $\mid$.

For instance,

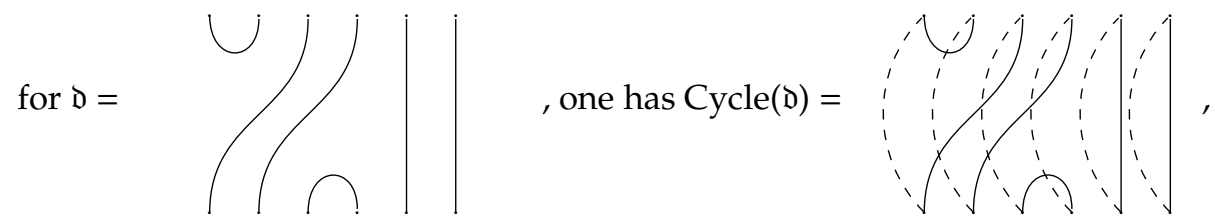

$\operatorname{ntriv}(\mathfrak{D})=1$, and $\operatorname{triv}(\mathfrak{D})=2$. Let $\operatorname{tr}$ denotes the trace computed by considering the hypermatrices as $N^{n} \times N^{n}$-matrices. Remark that $\operatorname{tr}\left(\mu\left(\mathfrak{D} \leftrightarrow \mathfrak{D}^{\prime}\right)\right)=\operatorname{tr}\left(\mu(\mathfrak{D}) \leftrightarrow \mu\left(\mathfrak{D}^{\prime}\right)\right)=\operatorname{tr}(\mu(\mathfrak{D})) \operatorname{tr}\left(\mu\left(\mathfrak{D}^{\prime}\right)\right)$ ( because the trace of a Kronecker product is the product of the trace). Hence, we deduce $\operatorname{tr}(\mu(\overbrace{|\ldots|}^{n \times}))=N^{n}$. We also have

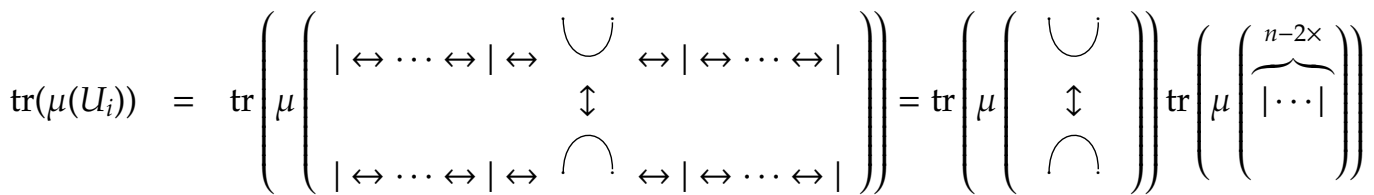

$$
\begin{aligned}
& =\operatorname{tr}\left(\begin{array}{c}
\bigcap \\
\mathfrak{\imath} \\
\bigcup
\end{array}\right) N^{n-2}=N^{n-2} d \text {. }
\end{aligned}
$$

In a similar way, one shows that for $|j-i|>1$ we have

$$
\operatorname{tr}\left(\mu\left(\begin{array}{c}
\mathfrak{U}_{i} \\
\uparrow \\
\mathfrak{U}_{j}
\end{array}\right)\right)=N^{n-4} d^{2}
$$

Furthermore, for any $i<n$ one has

$$
\operatorname{tr}\left(\mu\left(\mathfrak{U}_{i}\right)\right)=\operatorname{tr}\left(\mu\left(\begin{array}{c}
\mathfrak{U}_{i} \\
\mathfrak{1} \\
\mathfrak{U}_{i+1} \\
\mathfrak{1} \\
\mathfrak{U}_{i}
\end{array}\right)\right)=\operatorname{tr}\left(\mu\left(\begin{array}{c}
\mathfrak{U}_{i} \\
\mathfrak{i} \\
\mathfrak{U}_{i} \\
\mathfrak{\downarrow} \\
\mathfrak{U}_{i+1}
\end{array}\right)\right)=d \operatorname{tr}\left(\mu\left(\begin{array}{c}
\mathfrak{U}_{i} \\
\mathfrak{1} \\
\mathfrak{U}_{i+1}
\end{array}\right)\right) .
$$

And then

$$
\operatorname{tr}\left(\mu\left(\begin{array}{c}
\mathfrak{U}_{i} \\
\mathfrak{1} \\
\mathfrak{U}_{i+1}
\end{array}\right)\right)=\operatorname{tr}\left(\mu\left(\begin{array}{c}
\mathfrak{U}_{i+1} \\
\mathfrak{1} \\
\mathfrak{U}_{i}
\end{array}\right)\right)=\frac{1}{d} \operatorname{tr}\left(\mu\left(\mathfrak{U}_{i}\right)\right)=N^{n-2} .
$$

More generally, one conjectures

Conjecture 1. Let $\mu$ be a representation of $\mathcal{T}$ of dimension $N$. Then

$$
\operatorname{tr}(\mu(\mathfrak{D}))=N^{\operatorname{triv}(\mathfrak{D})} d^{\text {ntriv }(\mathfrak{D})},
$$

for any $\mathrm{D} \in T \ell_{n}$. 